\title{
Detection of VHE gamma-ray emission from the distant blazar 1ES 1101-232 with HESS and broadband characterisation
}

\author{
F. Aharonian ${ }^{1}$, A. G. Akhperjanian ${ }^{2}$, A. R. Bazer-Bachi ${ }^{3}$, M. Beilicke ${ }^{4}$, W. Benbow ${ }^{1}$, D. Berge ${ }^{1, \star}$, K. Bernlöhr ${ }^{1,5}$,
} C. Boisson ${ }^{6}$, O. Bolz ${ }^{1}$, V. Borrel ${ }^{3}$, I. Braun ${ }^{1}$, E. Brion ${ }^{7}$, A. M. Brown ${ }^{8}$, R. Bühler ${ }^{1}$, I. Büsching ${ }^{9}$, T. Boutelier ${ }^{17}$, S. Carrigan ${ }^{1}$, P. M. Chadwick ${ }^{8}$, L.-M. Chounet ${ }^{10}$, G. Coignet $^{11}$, R. Cornils ${ }^{4}$, L. Costamante ${ }^{1,23}$, B. Degrange ${ }^{10}$, H. J. Dickinson ${ }^{8}$, A. Djannati-Ataï ${ }^{12}$, L. O’C. Drury ${ }^{13}$, G. Dubus ${ }^{10}$, K. Egberts ${ }^{1}$, D. Emmanoulopoulos ${ }^{14}$, P. Espigat ${ }^{12}$, C. Farnier ${ }^{15}$, F. Feinstein ${ }^{15}$, E. Ferrero ${ }^{14}$, A. Fiasson ${ }^{15}$, G. Fontaine ${ }^{10}$, Seb. Funk ${ }^{5}$, S. Funk ${ }^{1}$, M. Füßling ${ }^{5}$, Y. A. Gallant ${ }^{15}$, B. Giebels ${ }^{10}$, J. F. Glicenstein ${ }^{7}$, B. Glück ${ }^{16}$, P. Goret ${ }^{7}$, C. Hadjichristidis ${ }^{8}$, D. Hauser ${ }^{1}$, M. Hauser ${ }^{14}$, G. Heinzelmann ${ }^{4}$, G. Henri ${ }^{17}$, G. Hermann ${ }^{1}$, J. A. Hinton ${ }^{1,14, \star \star}$, A. Hoffmann ${ }^{18}$, W. Hofmann ${ }^{1}$, M. Holleran ${ }^{9}$, S. Hoppe ${ }^{1}$, D. Horns ${ }^{18}$, A. Jacholkowska ${ }^{15}$, O. C. de Jager ${ }^{9}$, E. Kendziorra ${ }^{18}$, M. Kerschhaggl ${ }^{5}$, B. Khélifi ${ }^{10,1}$, Nu. Komin ${ }^{15}$, K. Kosack ${ }^{1}$, G. Lamanna ${ }^{11}$, I. J. Latham ${ }^{8}$, R. Le Gallou ${ }^{8}$, A. Lemière ${ }^{12}$, M. Lemoine-Goumard ${ }^{10}$, T. Lohse ${ }^{5}$, J. M. Martin ${ }^{6}$, O. Martineau-Huynh ${ }^{19}$, A. Marcowith ${ }^{3,15}$, C. Masterson ${ }^{1,23}$, G. Maurin ${ }^{12}$, T. J. L. McComb ${ }^{8}$, E. Moulin ${ }^{15,7}$, M. de Naurois ${ }^{19}$, D. Nedbal ${ }^{20}$, S. J. Nolan ${ }^{8}$, A. Noutsos ${ }^{8}$, J.-P. Olive ${ }^{3}$, K. J. Orford ${ }^{8}$, J. L. Osborne ${ }^{8}$, M. Panter ${ }^{1}$, G. Pelletier ${ }^{17}$, P.-O. Petrucci ${ }^{17}$, S. Pita ${ }^{12}$, G. Pühlhofer ${ }^{14}$, M. Punch ${ }^{12}$, S. Ranchon ${ }^{11}$, B. C. Raubenheimer ${ }^{9}$, M. Raue ${ }^{4}$, S. M. Rayner ${ }^{8}$, J. Ripken ${ }^{4}$, L. Rob ${ }^{20}$, L. Rolland ${ }^{7}$, S. Rosier-Lees ${ }^{11}$, G. Rowell ${ }^{1, \star \star \star}$, V. Sahakian ${ }^{2}$, A. Santangelo ${ }^{18}$, L. Saugé ${ }^{17}$, S. Schlenker ${ }^{5}$, R. Schlickeiser ${ }^{21}$, R. Schröder ${ }^{21}$, U. Schwanke ${ }^{5}$, S. Schwarzburg ${ }^{18}$, S. Schwemmer ${ }^{14}$, A. Shalchi ${ }^{21}$, H. Sol ${ }^{6}$, D. Spangler ${ }^{8}$, F. Spanier ${ }^{21}$, R. Steenkamp ${ }^{22}$, C. Stegmann ${ }^{16}$, G. Superina ${ }^{10}$, P. H. Tam ${ }^{14}$, J.-P. Tavernet ${ }^{19}$, R. Terrier ${ }^{12}$, M. Tluczykont ${ }^{10,23, \dagger}$, C. van Eldik ${ }^{1}$, G. Vasileiadis ${ }^{15}$, C. Venter ${ }^{9}$, J. P. Vialle ${ }^{11}$, P. Vincent ${ }^{19}$, H. J. Völk ${ }^{1}$, S. J. Wagner ${ }^{14}$, and M. Ward ${ }^{8}$

(Affiliations can be found after the references)

Received 4 January 2007 / Accepted 28 March 2007

\section{ABSTRACT}

Context. The blazar 1ES 1101-232 was observed with the High Energy Stereoscopic System (HESS) of Atmospheric Cherenkov Telescopes (ACT) in 2004 and 2005, for a live time of $43 \mathrm{~h}$. VHE $\left(E>10^{11} \mathrm{eV}\right) \gamma$-rays were detected for the first time from this object.

Aims. VHE observations of blazars are used to investigate the inner parts of the blazar jets, and also to study the extragalactic background light (EBL) in the near-infrared band.

Methods. Observations in 2005 were conducted in a multiwavelength campaign, together with the RXTE satellite and optical observations. In 2004, simultaneous observations with XMM-Newton were obtained.

Results. 1ES 1101-232 was detected with HESS with an excess of 649 photons, at a significance of $10 \sigma$. The measured VHE $\gamma$-ray flux amounts to $\mathrm{d} N / \mathrm{d} E=(5.63 \pm 0.89) \times 10^{-13}(E / \mathrm{TeV})^{-(2.94 \pm 0.20)} \mathrm{cm}^{-2} \mathrm{~s}^{-1} \mathrm{TeV}^{-1}$, above a spectral energy threshold of $225 \mathrm{GeV}$. No significant variation of the VHE $\gamma$-ray flux on any time scale was found. 1ES 1101-232 exhibits a very hard spectrum, and at a redshift of $z=0.186$, is the blazar with the highest confirmed redshift detected in VHE $\gamma$-rays so far.

Conclusions. The data allow the construction of truly simultaneous spectral energy distributions of the source, from the optical to the VHE band. Using an EBL model with $v F_{v}=14 \mathrm{nWm}^{-2} \mathrm{sr}^{-1}$ at $1.5 \mu \mathrm{m}$ as presented in Aharonian et al. (2006a) suggests an intrinsic VHE power output peak of the source at above $3 \mathrm{TeV}$.

Key words. gamma rays: observations - galaxies: active - BL Lacertae objects: individual: 1ES 1101-232

\section{Introduction}

Blazars (BL Lacs and Flat Spectrum Radio Quasars) are thought to be active galactic nuclei (AGN) that have their jet axis oriented close to the line of sight of the observer. The broadband

^ Now at CERN, Geneva, Switzerland.

$\star \star$ Now at School of Physics \& Astronomy, University of Leeds, Leeds LS2 9JT, UK.

$\star \star \star$ Now at School of Chemistry \& Physics, University of Adelaide, Adelaide 5005, Australia.

Now at DESY Zeuthen. spectral energy distribution (SED, $v F_{v}$ representation) of blazars is characterized by two peaks, one at optical to X-ray energies, and another at $\gamma$-ray energies. The low-energy branch is commonly explained as electron synchrotron emission. The highenergy branch can be explained in a variety of ways. In leptonic scenarios, it is assumed to result from Inverse Compton (IC) emission from the same electron population, up-scattering the self-generated synchrotron photons or external photons (synchrotron self-Compton, SSC, and external Compton, EC, e.g., Mushotzky 1977; Madejski \& Schwartz 1983; Ghisellini et al. 1985; Band \& Grindlay 1986; Dermer \& Schlickeiser 1993; 
Sikora et al. 1994). In alternative hadronic scenarios, accelerated protons are the main source of high-energy radiation, either directly or through the production of secondary particles (e.g., Biermann \& Strittmatter 1987; Aharonian 2000; Pohl \& Schlickeiser 2000; Mücke \& Protheroe 2001). Observationally, the SED and the variability in the different bands carry the information about the acceleration processes at work in the jet, and could ultimately also shed light on the energy transfer mechanism of the central engine - a supermassive black hole - into the jet.

Over the past fourteen years, VHE $\gamma$-ray emission from approximately a dozen blazars has been detected (see, e.g., Ong 2005, for a recent review). Both the detection of fast variability and the availability of broadband observations - especially including X-ray measurements - have been used to constrain individual source parameters (e.g., Krawczynski et al. 2002; Aharonian et al. 2005a). Increasing the number of known VHE blazars, especially at higher redshift, is of importance for two reasons:

(1) Relatively little is still known about the average behaviour of VHE blazars. Most VHE blazars detected so far belong to the classes of X-ray selected BL Lacs (XBL) or high-frequency peaked BL Lacs (HBL), but population studies are restricted by the low number of sources. Previous detections of VHE blazars have also been biased towards high states of the sources, because of the limited sensitivity of the available instruments. It was shown only recently that quiescent states can be detected now in short ( hours) observations (Aharonian et al. 2005a). Little is known about average activity cycles and flare time scales, except for a few sources: Mkn 421 (e.g., Aharonian et al. 2002b; Błażejowski et al. 2005), Mkn 501 (e.g., Aharonian et al. 1999; Krawczynski et al. 2002), and 1ES 1959+650 (e.g., Krawczynski et al. 2004; Albert et al. 2006).

(2) Source photons above $\sim 100 \mathrm{GeV}$ are attenuated by the EBL through $\gamma-\gamma$-interactions. Therefore, an EBL density in the relevant waveband range (typically $\sim 0.1$ to $\sim 10 \mu \mathrm{m}$ ) has to be assumed to derive the intrinsic blazar spectrum. Conversely, if it is possible to determine or constrain the intrinsic blazar spectrum through models, then constraints on the EBL density in the respective waveband can be obtained (e.g. Coppi \& Aharonian 1999; Aharonian et al. 2006a, hereafter AHA06a).

To date, 1ES 1101-232 is the most distant VHE blazar known with confirmed redshift $(z=0.186)$. It should be noted that the hard spectrum observed from 1ES 1101-232 and its relatively large redshift allow for strong constraints on the EBL density. This is described in detail in a separate paper (AHA06a).

In this paper, the discovery of VHE $\gamma$-ray emission from the blazar 1ES 1101-232 with HESS is reported. The paper is organized as follows: In Sect. 2, the source characteristics of 1ES 1101-232 are described. The results of the HESS observations of 1ES 1101-232 are presented in Sect. 3. In Sect. 4, multifrequency observations that were performed contemporaneously to the HESS observations are reported. In Sect. 5, we concentrate on the interpretation of the spectral energy distribution derived from the source.

\section{The HBL object 1ES 1101-232}

Emission from 1ES 1101-232 was first detected by the Ariel-5 X-ray satellite, the source A 1059-22 was however misidentified with the Abell 1146 cluster of galaxies at $z=0.139$ at that time (McHardy et al. 1981; Maccagni et al. 1978). The HEAO-1 source H 1101-232 was later correctly identified as a BL Lac type object, using the optical and radio counterparts (Buckley et al. 1985; Remillard et al. 1989). The source has been detected by multiple X-ray observatories, and for the purpose of this paper, the commonly used name 1ES 1101-232 from the Einstein slew survey is adopted (Elvis et al. 1992; Perlman et al. 1996).

1ES 1101-232 resides in an elliptical host galaxy at a redshift of $z=0.186$ (Remillard et al. 1989; Falomo et al. 1994). The host is presumably part of a galaxy cluster (Remillard et al. 1989; Pesce et al. 1994). VLA maps of the BL Lac show a one-sided diffuse structure to the north of $\sim 45^{\prime \prime}$ size, but no well-collimated jet outside a few kpc distance from the core (Laurent-Muehleisen et al. 1993). In the optical, the host galaxy is resolved (Remillard et al. 1989; Abraham et al. 1991). Falomo et al. (1993) deduced its brightness using a spectroscopic deconvolution of host and BL Lac. The most recent estimate of $m_{R}=16.41$ was derived from an angular profile fit (Falomo \& Ulrich 2000). The galaxy is one of the brightest BL Lac host galaxies so far detected, and also the only one known with significantly boxy isophotes (Falomo \& Ulrich 2000), indicating a merger process or extra dust components. The BL Lac itself has a typical brightness of $m_{V}=16-17$ (e.g., Remillard et al. 1989). The optical emission from 1ES 1101-232 has typically varied on the timescale of months (e.g., Remillard et al. 1989). Optical flares on intraday timescales have also been claimed in one observation (Romero et al. 1999).

The source has been classified earlier as an XBL (e.g., Scarpa \& Falomo 1997), and later on as an HBL (e.g., Donato et al. 2001), because of the dominance of synchrotron emission in the X-ray band. Several authors have concluded from the broadband characteristics of 1ES 1101-232 that this source is expected to emit VHE $\gamma$-ray emission at flux levels detectable by instruments like HESS (e.g., Wolter et al. 2000; Costamante \& Ghisellini 2002). Previous VHE observations with the Durham Mark 6 telescope in 1998 have only yielded flux limits (Chadwick et al. 1999). Also, in the GeV $\gamma$-ray domain, EGRET did not detect emission from 1ES 1101-232 (Lin et al. 1996).

In previously published SSC models (Wolter et al. 2000; Costamante \& Ghisellini 2002), the IC peak was generally expected to be around $100 \mathrm{GeV}$, but this seems not to be the case as shown in this paper.

\section{HESS observations of 1ES1101-232}

Observations were made with the HESS Cherenkov telescopes in April and June 2004, and in March 2005. On June 8th 2004, also XMM-Newton X-ray observations were obtained, scheduled such that simultaneous HESS observations could be conducted. Following the detection of a weak signal in the 2004 HESS observations, an extended multifrequency campaign was organized for 11 nights in March 2005, to study the broadband emission from 1ES 1101-232 and to search for (possibly correlated) variability in the different wavebands. Simultaneous observations were carried out with HESS, X-ray measurements with RXTE, and optical measurements with the ROTSE $3 \mathrm{c}$ robotic telescope.

\subsection{The HESS experiment}

The HESS experiment (Hinton et al. 2004) consists in phase I of four ACTs operating stereoscopically. Each telescope consists of 
a tesselated $13 \mathrm{~m}$ diameter (107 $\mathrm{m}^{2}$ surface area) mirror, which focuses the Cherenkov light from the showers of secondary particles created by the interaction of $\gamma$-rays in the atmosphere onto a camera in the focal plane. Each camera consists of 960 photomultipliers with a pixel size of $0.16^{\circ}$, providing a field of view (FoV) of $5^{\circ}$. The array is located in the Khomas highlands in Namibia $\left(-23^{\circ} 16^{\prime}, 16^{\circ} 30^{\prime}, 1835 \mathrm{~m}\right.$ a.s.l.).

The angular resolution of the stereo system is better than $0.1^{\circ}$ per event. The energy threshold of HESS is about $100 \mathrm{GeV}$ (at zenith), with spectral measurements possible above $\sim 150 \mathrm{GeV}$ with an energy resolution of $15 \%$. The energy threshold increases with zenith angle. For the data set of 1ES 1101-232 discussed in this paper, the time-averaged spectrum presented in this paper has an energy threshold of $225 \mathrm{GeV}$.

The minimum detectable point source flux above $100 \mathrm{GeV}$ with HESS is $\sim 4 \times 10^{-12} \mathrm{erg} \mathrm{cm}^{-2} \mathrm{~s}^{-1}$ for a $5 \sigma$ detection in $25 \mathrm{~h}$, corresponding to $\sim 10 \mathrm{mCrab}$ (Aharonian et al. 2006c). The sensitivity enabled a $\geq 3 \sigma$ detection per night $(\sim 5 \mathrm{~h})$ in the case of the 1ES 1101-232 observations.

\subsection{HESS observations of 1ES 1101-232}

First observations of 1ES 1101-232 with HESS were performed during four nights in April 2004, for a total live time of $2.7 \mathrm{~h}$ after quality selection, and for six nights in June 2004, for a total of $8.4 \mathrm{~h}$ after quality selection. The total data set in March 2005 after quality selection amounts to $31.6 \mathrm{~h}$ live time. The observation $\log$ is shown in Table 1.

HESS observations were taken in runs of typically $28 \mathrm{~min}$ each. Runs were taken in wobble mode ${ }^{1}$, with a declination or right ascension offset of $\pm 0.5^{\circ}$ in 2004 and $\pm 0.7^{\circ}$ in 2005 . Since the $\gamma$-ray acceptance of the HESS instrument is nearly constant within $1^{\circ}$ radius from the FoV center, this change of observation mode results in a slight sensitivity increase, as a larger background control area with equal acceptance in the FoV can be used.

The data cleaning to derive a set of good quality runs, which are used in the data analysis, consists of two filtering processes. First, from the individual shower images as recorded from triggered telescopes, all pixels that have not worked properly are removed; occasionally, entire cameras have to be excluded from individual runs. Then runs are discarded that show a too low or fluctuating event trigger rate, caused by bad atmospheric conditions or clouds.

\subsection{Analysis of the HESS data}

The HESS data have been processed and analysed according to the standard analysis chain as detailed for instance in Aharonian et al. (2005b) and Benbow (2005). Shower images as recorded by individual telescopes are parametrized using the Hillas parametrisation. The shower direction (i.e., the origin of the incoming $\gamma$-ray) and the projected shower impact point on the ground are determined from a stereoscopic reconstruction of the air shower. A large fraction of the background is suppressed using cuts on the shower shape parameters mean reduced scaled width $m r s w$ and length $m r s l$. Photon directions are used for a one-dimensional projection including background estimate ( $\theta^{2}$-plot, Fig. 1) and to reconstruct the source location (Fig. 2). The background after shape cuts is estimated from different

${ }^{1}$ In wobble mode, the source is displaced with respect to the center of the FoV, the sign of the offset alternating between consecutive runs. This permitted continuous monitoring of 1ES 1101-232.
Table 1. Log of the HESS observations on 1ES 1101-232 in 2004 and 2005. Numbers reflect the observations after data cleaning and good run selection. Nights with observations performed on 1ES 1101-232, where however all HESS observations needed to be discarded because of weather selections, are marked with a "“".

\begin{tabular}{l|l|l|l|l|l}
\hline \hline Period & $\mathrm{MJD}^{1}$ & $T^{2}$ (runs) & $F^{3}$ & $S^{4}$ & X-ray \\
\hline Apr. & 53111 & $0.91(3)$ & $1.1_{1.1}^{+2.6}$ & 1.63 & \\
2004 & 53113 & $0.71(2)$ & $6.5_{3.6}^{+3.7}$ & 0.90 & \\
& 53115 & $0.83(2)$ & $3.0_{2.7}^{+2.7}$ & 0.33 & \\
& 53117 & $0.21(1)$ & $5.7_{4.5}^{+6.4}$ & 1.82 & \\
\hline Jun. & 53162 & $0.85(2)$ & $<4.6^{5}$ & 0.41 & \\
2004 & 53163 & - & & & \\
& 53164 & - & & & \\
& 53165 & $3.18(7)$ & $5.5_{2.6}^{+2.6}$ & 3.20 & XMM \\
& 53166 & $2.72(6)$ & $8.2_{2.8}^{+2.8}$ & 3.36 & \\
& 53167 & $1.72(5)$ & $4.6_{2.4}^{+2.4}$ & 1.81 & \\
\hline Mar. & 53435 & - & & & XTE \\
& 53436 & $5.15(11)$ & $7.7_{3.6}^{+3.6}$ & 5.63 & XTE \\
& 53437 & $5.29(12)$ & $2.1_{1.8}^{+3.1}$ & 2.87 & XTE \\
& 53438 & $5.12(10)$ & $5.4_{1.7}^{+1.7}$ & 5.00 & XTE \\
& 53439 & $5.01(10)$ & $4.6_{1.6}^{+1.6}$ & 3.39 & XTE \\
& 53440 & $3.25(7)$ & $4.2_{2.1}^{+2.1}$ & 3.10 & XTE \\
& 53441 & $1.65(3)$ & $1.6_{-1.6}^{+2.7}$ & 2.16 & XTE \\
& 53442 & - & & & XTE \\
& 53443 & $2.42(5)$ & $5.0_{2.0}^{+2.0}$ & 2.47 & XTE \\
& 53444 & $1.80(4)$ & $8.0_{2.6}^{+2.6}$ & 3.59 & XTE \\
& 53445 & $1.92(4)$ & $2.4_{1.6}^{+1.9}$ & 1.90 & XTE \\
\hline
\end{tabular}

${ }^{1}$ Modified Julian date. ${ }^{2}$ Live time $T[\mathrm{~h}] .{ }^{3}$ Flux $F(E>200 \mathrm{GeV})$ $\left[10^{-12} \mathrm{erg} \mathrm{cm}^{-2} \mathrm{~s}^{-1}\right] .{ }^{4}$ Detection significance $S$ in units of standard deviations. ${ }^{5}$ Upper limit at $99 \%$ confidence level.

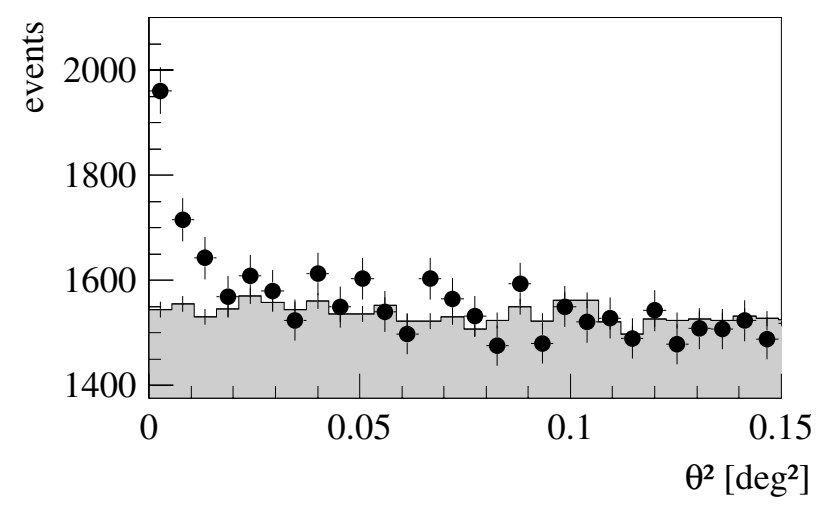

Fig. 1. Angular event distribution. Events are from the entire HESS data set on 1ES 1101-232, after shape cuts to suppress a large fraction of the background. The filled circles denote the event distribution in squared distance with respect to the direction to 1ES 1101-232. The filled histogram represents the expected background. For this histogram, the distributions obtained from seven control regions were summed and renormalized.

control regions in the FoV, having the same distance to the center of the FoV in order to be independent of the radial acceptance change (Hinton et al. 2005). For this data set, a background region with an area $A_{\text {off }} 11$ (in 2004) or 15 (in 2005) times larger than the on-source area $A_{\text {on }}$ was used. The applied standard cuts (see Table 2) were optimized on a simulated source with $10 \%$ of the flux from the Crab Nebula and a Crab-like power-law spectrum $\propto E^{-\Gamma}$ with $\Gamma=2.6$. 
Table 2. HESS analysis parameters and event numbers. Values are given for the total HESS data set as well as for the three data subsets considered.

\begin{tabular}{|c|c|}
\hline \multicolumn{2}{|l|}{ Cuts } \\
\hline image distance to FoV center & $<2$ deg \\
\hline image amplitude & $>80$ ph.e. \\
\hline number of telescopes & $\geq 2$ \\
\hline cut on shower width & $-2.0<m r s w<0.9$ \\
\hline cut on shower length & $-2.0<m r s l<2.0$ \\
\hline distance to source $\theta^{2}$ & $<0.0125 \mathrm{deg}^{2}$ \\
\hline \multicolumn{2}{|c|}{ Total Data Set (April 2004, June 2004, March 2005) } \\
\hline$N_{\text {on }}$ (events) & 4276 \\
\hline$N_{\text {off }}$ (events) & 54345 \\
\hline normalisation $\alpha=A_{\text {on }} / A_{\text {off }}$ & 0.0667 \\
\hline excess (events) & 649.0 \\
\hline significance & $10.1 \sigma$ \\
\hline \multicolumn{2}{|l|}{ March 2005 Data Set } \\
\hline$N_{\text {on }}$ (events) & 3028 \\
\hline$N_{\text {off }}$ (events) & 42427 \\
\hline normalisation $\alpha=A_{\text {on }} / A_{\text {off }}$ & 0.0597 \\
\hline excess (events) & 495.8 \\
\hline significance & $9.3 \sigma$ \\
\hline \multicolumn{2}{|l|}{ June 2004 Data Set } \\
\hline$N_{\text {on }}$ (events) & 902 \\
\hline$N_{\text {off }}$ (events) & 8531 \\
\hline normalisation $\alpha=A_{\mathrm{on}} / A_{\mathrm{off}}$ & 0.0926 \\
\hline excess (events) & 112.4 \\
\hline significance & $3.7 \sigma$ \\
\hline \multicolumn{2}{|l|}{ April 2004 Data Set } \\
\hline$N_{\text {on }}$ (events) & 346 \\
\hline$N_{\text {off }}$ (events) & 3405 \\
\hline normalisation $\alpha=A_{\text {on }} / A_{\text {off }}$ & 0.09091 \\
\hline excess (events) & 36.5 \\
\hline significance & $1.9 \sigma$ \\
\hline
\end{tabular}

Energy estimates for individual photons are based on the comparison of the measured image amplitudes to data from simulated events, using the measured shower core position and zenith angle as parameters. The resolution per event is $\sim 15 \%$ above the spectral threshold, which is defined as the energy threshold above which the energy reconstruction bias is less than $10 \%$. Only events above this safe threshold are used to compute energy spectra. The effective area is based on Monte Carlo simulations and is depending on zenith angle and system configuration (i.e., which telescopes are included in a particular run).

Compared to the analysis results used in AHA06a, an improved energy calibration of the telescope system was applied to the data, better taking into account the long-term optical sensitivity changes of the instrument (Aharonian et al. 2006c). For the given total data sample, this energy scale recalibration yields a safe energy threshold of $225 \mathrm{GeV}$ (compared to $165 \mathrm{GeV}$ used in AHA06a) and a flux normalisation increase of $27 \%$ at $1 \mathrm{TeV}$. After this correction, the systematic flux uncertainty is now estimated as 20\% (Aharonian et al. 2006c). Reconstructed spectral indices were not affected significantly by these calibration updates, the systematic error estimate for reconstructed photon indices is $\Delta \Gamma_{\text {sys }} \sim 0.1$ (AHA06a; Aharonian et al. 2006c). The recalibration slightly increased the background noise in this data set which therefore has an excess significance of $10.1 \sigma$, slightly smaller than the detection significance of $11.7 \sigma$ that was derived from the original data set used in AHA06a.

The results derived have been verified using a completely independent calibration and analysis chain, which is described for instance in Aharonian et al. (2006b) and Lemoine et al. (2006).

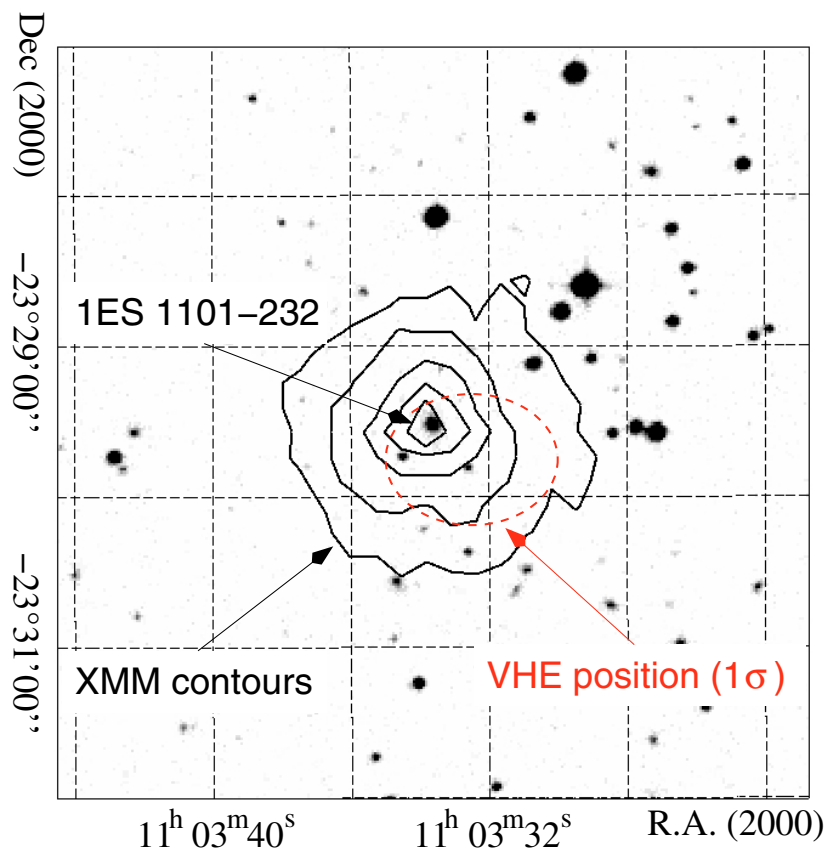

Fig. 2. Sky map of the region surrounding 1ES 1101-232. An $R$-band image made by the Anglo-Australian observatory with the UK Schmidt telescope is shown in grey-scale. The host galaxy (labeled 1ES 1101-232 in the image) of the BL Lac is resolved as an elliptical galaxy, with boxy isophotes at larger radii (Falomo \& Ulrich 2000). The dashed ellipse denotes the $1 \sigma$ error of the reconstructed VHE $\gamma$-ray source position. The solid contours are from the XMM-Newton X-ray measurements with the MOS 2 camera, as discussed in Sect. 4.2. The contour spacing is logarithmic $(0.1,0.3,1.4,5.8$ and $24 \%$ of the peak intensity), the outermost contour also denotes the edge of the effective MOS $2 \mathrm{FoV}$ for this small window mode observation.

\subsection{Skymap and identification of the VHE $\gamma$-ray source}

Source confusion is generally a minor issue in VHE data, especially from extragalactic sources. No other source near 1ES $1101-232\left(11^{\mathrm{h}} 03^{\mathrm{m}} 37.57^{\mathrm{s}}, \quad-23^{\circ} 29^{\prime} 30.2^{\prime \prime}, \quad \mathrm{J} 2000\right.$, Laurent-Muehleisen et al. 1993) is a good candidate for VHE $\gamma$-ray emission. The VHE $\gamma$-ray source location was derived from the skymap of VHE photons and is $11^{\mathrm{h}} 03^{\mathrm{m}} 36.5^{\mathrm{s}} \pm$ $2.5_{\text {stat }}^{\mathrm{s}},-23^{\circ} 29^{\prime} 45^{\prime \prime} \pm 26_{\text {stat }}^{\prime \prime}(\mathrm{J} 2000)$, which is consistent with the above given radio position, and also with the optical and X-ray positions as shown in Fig. 2. As the present VHE data do not exhibit variability, the identification of the VHE $\gamma$-ray source is based on its location and the interpretation of the SED.

Figure 2 also shows that the X-ray imaging data, which were obtained with XMM-Newton, are compatible with the XMM-Newton point spread function. This excludes the influence of possible nearby X-ray sources in the FoV of nonimaging X-ray measurements such as RXTE.

\subsection{VHE $\gamma$-ray light curve}

The fluxes derived from the three HESS data sets (April 2004, June 2004, March 2005) are statistically compatible with each other, indicating that the VHE $\gamma$-ray flux has remained constant throughout these observing periods. However, as the detections from the April $(1.9 \sigma)$ and June $2004(3.7 \sigma)$ data sets alone are not very significant, only flux variations of a factor of approximately two or larger can be excluded. Night-by-night variations were also searched for, but the corresponding light curve is 


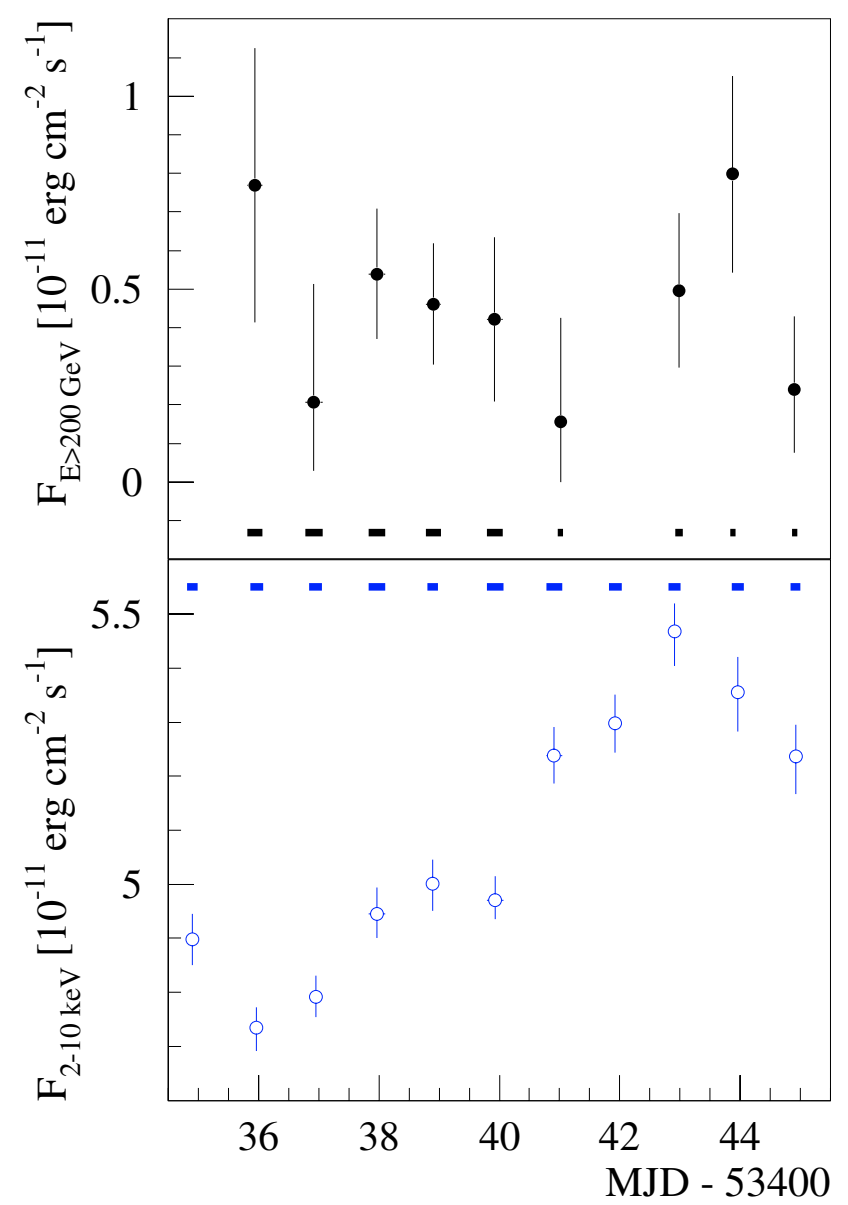

Fig. 3. March 2005 light curve of 1ES 1101-232. MJD = 53435 corresponds to the night Mar. 5.-6. Upper panel: VHE $\gamma$-ray flux as measured with HESS, in nightly averaged bins. Within errors, the flux was constant. Lower panel: X-ray flux $(2-10 \mathrm{keV})$, derived from the PCU 2 detector onboard RXTE. Note that the flux scale is truncated, the $\mathrm{X}$-ray flux difference from minimum to maximum is $\sim 15 \%$. The thick horizontal bars in both panels denote the times (first to last measurement of an observing night) when the VHE and X-ray data were taken, indicating the high degree of simultaneity of the two data sets.

compatible with a constant flux (probability for a constant flux of the total data set $85 \%$, of the March 2005 data set $64 \%$ ).

In Fig. 3, the nightly averaged light curve of HESS is shown for the March 2005 period, together with simultaneous X-ray measurements from the RXTE satellite. The VHE $\gamma$-ray flux is given in integral flux units above $200 \mathrm{GeV}$ and was computed under the assumption of the time-averaged spectral index of $\Gamma=2.94$. The X-ray fluxes were similarly derived, details are given in Sect. 4.1.1. The RXTE data indicate an X-ray flux variation of $\sim 15 \%$ (min-max), whereas the simultaneously taken HESS data are not sensitive enough to detect possible correlations with similar amplitudes in the X-ray and VHE bands.

\subsection{VHE $\gamma$-ray spectrum}

The differential energy spectra derived for the entire HESS data set, as well as for the June 2004 and the March 2005 data subsets, are shown in the left panels of Fig. 4. The low statistics of the April 2004 data set prevented us from performing a spectral analysis on that subset. The measured spectra are compatible with power laws, Table 3 lists the corresponding photon indices $\Gamma_{\mathrm{abs}}$ derived from fits between 0.2 and $4 \mathrm{TeV}$.
The fit for the total spectrum gives $\mathrm{d} N / \mathrm{d} E=(5.63 \pm 0.89) \times$ $10^{-13}(E / \mathrm{TeV})^{-(2.94 \pm 0.20)} \mathrm{cm}^{-2} \mathrm{~s}^{-1} \mathrm{TeV}^{-1}$ above a spectral energy threshold of $225 \mathrm{GeV}$. The integral flux is $F(E>200 \mathrm{GeV})=$ $(4.5 \pm 1.2) \times 10^{-12} \mathrm{erg} \mathrm{cm}^{-2} \mathrm{~s}^{-1}$.

Spectral bins above $4 \mathrm{TeV}$ do not contain a significant flux detection. Therefore, from these bins $99 \%$ upper limits were derived and shown in Fig. 4. The photon indices $\Gamma_{\text {abs }}$ were derived excluding these bins. Table 3 additionally lists photon indices $\Gamma_{\text {abs }}^{*}$ from single power law fits, for which these flux estimates above $4 \mathrm{TeV}$ were included in $\chi^{2}$-fits. This is a viable procedure since the error bars are dominated by background estimates and are therefore mostly Gaussian. However, the results depend on the assumption that the power laws would extend to these high energies.

\subsection{VHE spectrum corrected for EBL absorption}

In the following, the data sets were corrected for EBL absorption, to investigate whether the intrinsic spectra show evidence for a cut-off towards high energies. Spectral changes between periods were also searched for. The deabsorbed spectra are used in the SED, as discussed in Sect. 5.

The intrinsic spectrum of 1ES 1101-232 depends on the assumed EBL spectrum. For a detailed discussion about the EBL absorption of VHE $\gamma$-ray spectra, we refer the reader to, e.g., Aharonian (2001). Corrections with a range of plausible EBL spectra result in deabsorbed spectra of 1ES 1101-232 that can be described over the entire detected energy range, i.e. between 0.2 and $4 \mathrm{TeV}$, by a single power law of photon in$\operatorname{dex} \Gamma_{\text {deabs }}$, i.e., $\Gamma_{\text {deabs }}=\Gamma_{\text {abs }}-\Delta \Gamma$, see AHA06a; Stecker \& Scully (2006).

\subsubsection{Maximum EBL}

To represent what AHA06a considered as the highest possible EBL level, the EBL shape P0.45 from AHA06a was used (cf. also Sect. 3.8). This shape reflects an EBL level of $14 \mathrm{nWm}^{-2} \mathrm{sr}^{-1}$ at $1.5 \mu \mathrm{m}$ after scaling down by $15 \%$ to take galaxy evolution effects into account. The such deabsorbed spectra of 1ES 1101-232 are shown in the right panels of Fig. 4. The fit to the deabsorbed spectrum of the total data set in the energy range 0.2 to $4 \mathrm{TeV}$ yields $\Gamma_{\text {deabs }}=1.51 \pm 0.17$. The spectra of the two subsets considered $\left(\Gamma_{\text {Jun. } 2004}=1.70 \pm 0.47\right.$ and $\left.\Gamma_{\text {Mar. } 2005}=1.49 \pm 0.19\right)$ are statistically compatible with each other.

Similar results are obtained when using the EBL "baseline" model described in Stecker et al. (2006), as shown by Stecker \& Scully (2006). Also the EBL model by Primack et al. (2001) yields similar numbers, after a slight reduction of $15 \%$ to match the above quoted level of $14 \mathrm{nWm}^{-2} \mathrm{sr}^{-1}$.

It is of interest to test whether the upper limits above $4 \mathrm{TeV}$ are compatible with a power-law extrapolation of the lower energy spectra, or are indicative of a steepening of the spectrum. For the intrinsic spectra, this would imply an observational hint for a peak in the SED at that energy range. For the deabsorbed spectra, only those bins above $4 \mathrm{TeV}$ could be included where the chosen EBL parametrisation $(P 0.45)$ can still be considered valid, which restricts the range to $\leq 7 \mathrm{TeV}$. As justified in Sect. 3.6, $\chi^{2}$-tests were applied. For example, for the March 2005 spectrum, the probability of the spectrum being compatible with $\Gamma=1.49$ changes from $65 \%$ to $49 \%$. Hence, there is no observational hint for a steepening of the spectrum above $\sim 4 \mathrm{TeV}$, but the data do not exclude a spectral change above that energy 


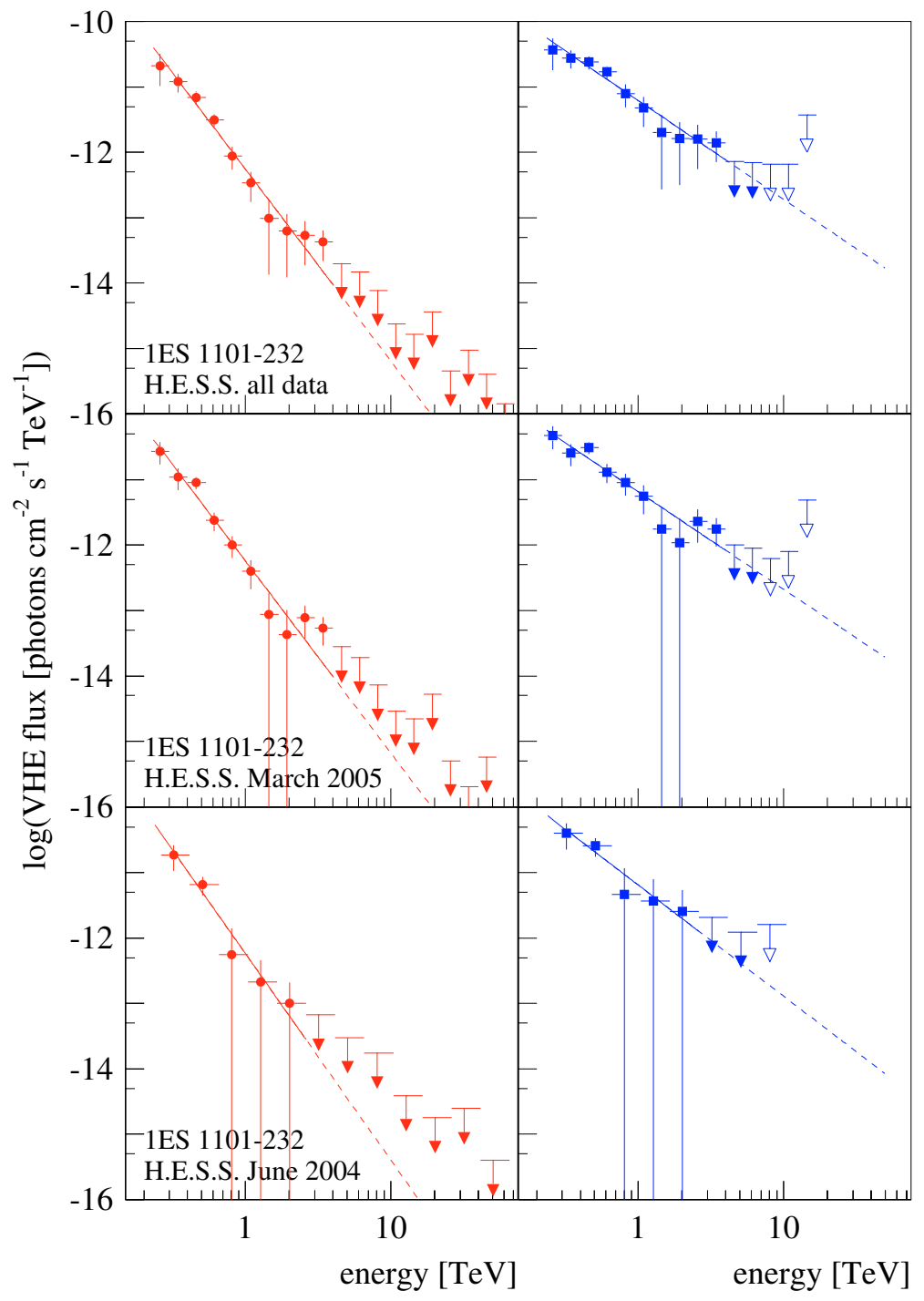

Fig. 4. VHE $\gamma$-ray spectra from 1ES 1101-232. Left panels: reconstructed photon flux, as measured with HESS. Right panels: photon spectra after correction for maximum EBL absorption, using an EBL model with $14 \mathrm{nWm}^{-2} \mathrm{sr}^{-1}$ at $1.5 \mu \mathrm{m}$ as described in Sect. 3.7.1. Upper limits in these deabsorbed spectra at energies above $7 \mathrm{TeV}$ are shown as open symbols only, because of strong EBL uncertainties at these high energies. In all panels, solid lines denote power-law fits between 0.2 and $4 \mathrm{TeV}$. Extrapolations of these power laws to higher energies are shown as dashed lines.

Table 3. Photon indices from power-law fits to the VHE spectra of 1ES 1101-232. $\Gamma_{\text {abs }}$ and $\Gamma_{\text {abs }}^{*}$ are from fits to the measured spectra, $\Gamma_{\text {deabs }}$ and $\Gamma_{\text {deabs }}^{*}$ from fits to the deabsorbed spectra as described in Sect. 3.7.1. $\Gamma_{\text {abs }}$ and $\Gamma_{\text {deabs }}$ correspond to the fits shown as solid lines in Fig. 4. Photon indices $\Gamma_{\mathrm{abs}}^{*}$ and $\Gamma_{\text {deabs }}^{*}$ are from fits including spectral bins above $4 \mathrm{TeV}$, which are compatible with zero flux, under the assumption that the power laws extend to these high energies.

\begin{tabular}{lcccc}
\hline \hline & $\Gamma_{\text {abs }}$ & $\Gamma_{\text {abs }}^{*}$ & $\Gamma_{\text {deabs }}$ & $\Gamma_{\text {deabs }}^{*}$ \\
& $0.23-4.0 \mathrm{TeV}$ & $0.23-16.9 \mathrm{TeV}$ & $0.23-4.0 \mathrm{TeV}$ & $0.23-7.1 \mathrm{TeV}$ \\
\hline All data & $2.94_{-0.21}^{+0.20}$ & $3.10_{-0.19}^{+0.17}$ & $1.51_{-0.19}^{+0.17}$ & $1.74_{-0.17}^{+0.15}$ \\
March 2005 & $2.94_{-0.23}^{+0.21}$ & $3.08_{-0.21}^{+0.18}$ & $1.49_{-0.20}^{+0.19}$ & $1.68_{-0.18}^{+0.16}$ \\
June 2004 & $3.16_{-0.61}^{+0.48}$ & $3.45_{-0.59}^{+0.41}$ & $1.70_{-0.61}^{+0.47}$ & $2.19_{-0.60}^{+0.40}$ \\
\hline
\end{tabular}

either. Photon indices $\Gamma_{\text {deabs }}^{*}$ from power-law fits in the energy range 0.2 to $7 \mathrm{TeV}$ are listed in Table 3 .

\subsubsection{Further EBL levels}

Lowering the EBL level used for the deabsorbtion leads to a softening of the spectra. Lower limits in the relevant EBL waveband range come from galaxy counts (Madau \& Pozzetti 2000) and are of the order of $10 \mathrm{nWm}^{-2} \mathrm{sr}^{-1}$ at $1.5 \mu \mathrm{m}$. The resolved galaxy counts may however represent a too low EBL level because of possibly missed light (Totani et al. 2001). Nevertheless, to represent the such constrained minimum EBL, the representation P0.40 (Aharonian et al. 2006a) was chosen and scaled down by $15 \%$ to take galaxy evolution effects into account. Using this minimum EBL to deabsorb the spectra result in $\Gamma_{\text {deabs }}=1.85 \pm$ $0.18, \Gamma_{\text {Jun. } 2004}=2.05 \pm 0.56$ and $\Gamma_{\text {Mar. } 2005}=1.84 \pm 0.20$.

EBL models higher than the maximum EBL level were described, e.g., by Stecker et al. (2006, "fast evolution" case) and 
by Kneiske et al. (2004, "best fit" model), with an EBL density of about $v F_{v}(1.5 \mu \mathrm{m}) \simeq 20 \mathrm{nWm}^{-2} \mathrm{sr}^{-1}$. As shown in Stecker \& Scully (2006), the "fast evolution" EBL would lead to an intrinsic spectrum with $\Gamma_{\text {deabs }} \simeq 1.0$. This result would be in conflict with the assumption of a limit on the intrinsic hardness of VHE blazar spectra, see next section and Sect. 5.1.

\subsection{EBL limit}

Following the discussion in AHA06a, we assume that the intrinsic blazar spectrum did not have a photon index significantly harder than 1.5 (i.e., $\Gamma_{\text {deabs }}<1.5$ ), taking the present observational and theoretical knowledge of VHE blazar spectra into account. Applied to the data from 1ES 1101-232, this limit results in an upper limit of the EBL density in the waveband range of $0.75-3.5 \mu \mathrm{m}$, with a peak density of $(14 \pm 4) \mathrm{nWm}^{-2} \mathrm{sr}^{-1}$ at $1.5 \mu \mathrm{m}$ for typical EBL shapes as reported in AHA06a.

Given that the updated calibration of the data set (Sect. 3.3) involves a change of the energy calibration which is slightly larger than the systematic error of $\pm 15 \%$ quoted in AHA06a, the procedure described in that paper to derive the EBL upper limit was repeated. The updated analysis constrains the total spectrum to a power law with $\Gamma=2.94$ between 0.23 and $4.0 \mathrm{TeV}$, whereas in AHA06a $\Gamma=2.88$ between 0.16 and $3.3 \mathrm{TeV}$ was used. The EBL limit derived with these updated numbers differs from the value in AHA06a by less than 1\%, well below any statistical uncertainties involved. The upper limit for the EBL spectrum as given above is therefore confirmed.

The error quoted for the peak density mostly comes from the statistical error of the photon index derived from the 1ES 1101-232 data set. This uncertainty is therefore well represented by the statistical errors of the deabsorbed spectra of 1ES 1101-232 discussed in the previous section. It is therefore inappropriate to translate the value of $(14 \pm 4) \mathrm{nWm}^{-2} \mathrm{sr}^{-1}$ into an $68 \%$ or $95 \%$ upper limit. We remark that - because of the procedure described above - the choice of $14 \mathrm{nWm}^{-2} \mathrm{sr}^{-1}$ yields by construction an intrinsic photon index of the total 1ES 1101-232 data set of 1.5.

\section{Broadband observations of 1ES 1101-232}

\subsection{The observation campaign in March 2005}

\subsubsection{X-ray data}

110.2 ks RXTE observation time for monitoring observations have been obtained, which were scheduled such that simultaneous HESS observations were possible for 11 consecutive nights in March 2005 (see Table 1). Because of Earth occultation and downtime in the southern Atlantic anomaly (SAA), the average on-time of RXTE was $56 \%$ during the "nightly" satellite observation windows.

RXTE data were analyzed using standard reduction routines. During most observations, two PCA detectors (PCU 0 and PCU 2) were active. For the analysis presented here, only results from PCU 2 were used. PCU 0 has lost its front veto layer, and is therefore susceptible to unmodeled and unremoved background events (Xue \& Cui 2005). Only the signal from the top layer $(\mathrm{X} 1 \mathrm{~L}, \mathrm{X} 1 \mathrm{R})$ was used for optimum signal to noise ratio. The STANDARD2 data were extracted using XDF and processed using ftools (Blackburn 1995) from HEASOFT 6.0.4. The data were filtered using standard criteria recommended by the RXTE guest observer facility (avoidance of Earth's limb, SAA, and tracking offsets). Electron contamination was suppressed by limiting the corresponding ELECTRON2 parameter to below 0.1 . The effective exposure after all screening was $89.6 \mathrm{ks}$. Background data were parametrized using pcabackest v3.0, using the faint background model. Response matrices were created with pcarsp v10.1, and nightly and total spectra were extracted with saextrct $\mathrm{v} 4.2 \mathrm{~d}$. Spectral fitting was performed with XSPEC v11.3.1, using PCA channels 5-32 (corresponding approximately to 3-15 keV). To account for Galactic absorption, a column density of $N_{\mathrm{H}}=5.76 \times 10^{20} \mathrm{~cm}^{-2}$ was used in the spectral fitting (cf., e.g., Wolter et al. (1998), and also the PIMMS nH program). The influence of $N_{\mathrm{H}}$ is however marginal at this energy band.

No flux variability was found within any of the 11 observing nights. Between nightly averages, moderate changes were observed (the probability for a constant flux is $10^{-5}$, using the nightly averaged count rates). No hint for spectral variability was found. The light curve shown in Fig. 3 was derived by fixing the spectral model to the broken power law derived for the total spectrum (see next paragraph), while leaving the normalisation as the only free parameter. Within statistical errors, the nightly fluxes derived are compatible with fluxes derived from single power-law fits with two free parameters (slope and normalisation). We conclude that 1ES 1101-232 was probably in a non-flaring state during this observation campaign. The simultaneous HESS measurements are not sensitive to VHE $\gamma$-ray flux changes of similar variability amplitudes.

As no spectral variability and only moderate flux changes were observed, a single averaged energy spectrum for the entire data set was derived. The spectrum between 3 and $15 \mathrm{keV}$ is incompatible with a pure power law $\left(\chi_{\text {red }}^{2}=2.08\right.$ for 26 d.o.f., nullhypothesis probability ${ }^{2} 10^{-3}$ ), but a broken power law yields an acceptable fit with $\chi_{\text {red }}^{2}=1.18$ (24 d.o.f., null-hypothesis probability $25 \%$ ). The total unabsorbed flux is $F_{2-10 \mathrm{keV}}=$ $\left(5.07 \pm 0.02_{\text {stat }}\right) \times 10^{-11} \mathrm{erg} \mathrm{cm}^{-2} \mathrm{~s}^{-1}$. In Fig. 5, the unfolded spectrum is shown. The spectrum is quite soft, with a photon index of $\Gamma=2.49 \pm 0.02_{\text {stat }}$ below the break energy $E_{\text {break }}=(7.9 \pm$ 1.0) $\mathrm{keV}$, and a slight softening to $\Gamma=2.78_{-0.11}^{+0.16}$ above $E_{\text {break }}$. The spectrum shows that the peak in the SED was below $\sim 3 \mathrm{keV}$ during the observations.

Possible systematic errors of the PCU spectrum were investigated by comparing recent archived spectra from Cassiopeia A with data from previous years, see the recommendations in the RXTE frequently asked questions ${ }^{3}$. For the purpose of the analysis presented here, we concluded that systematic errors are of no concern.

For the construction of the simultaneous SED discussed in Sect. 5, the X-ray spectrum derived from the entire RXTE data set was used, and similarly the HESS spectrum from the entire March 2005 data set. A restriction of both the HESS and RXTE data to the strictly simultaneous times appears overcautious, given the steady measured fluxes, and would have reduced the statistical accuracy, since only $13.3 \mathrm{~h}(54 \%)$ of 24.9h RXTE observations are overlapping with HESS data. These $13.3 \mathrm{~h}$ conversely represent $37 \%$ of the total March 2005 HESS data set (35.6h of on-source observation time). We note that the March 2005 data set presents - in terms of simultaneity and statistical accuracy - the best VHE/X-ray data set for 1ES 1101-232 so far.

\footnotetext{
2 i.e., the probability that the assumed function fits the data.

3 http://heasarc.gsfc.nasa.gov/docs/xte/ftools/ xtefaq.html
} 


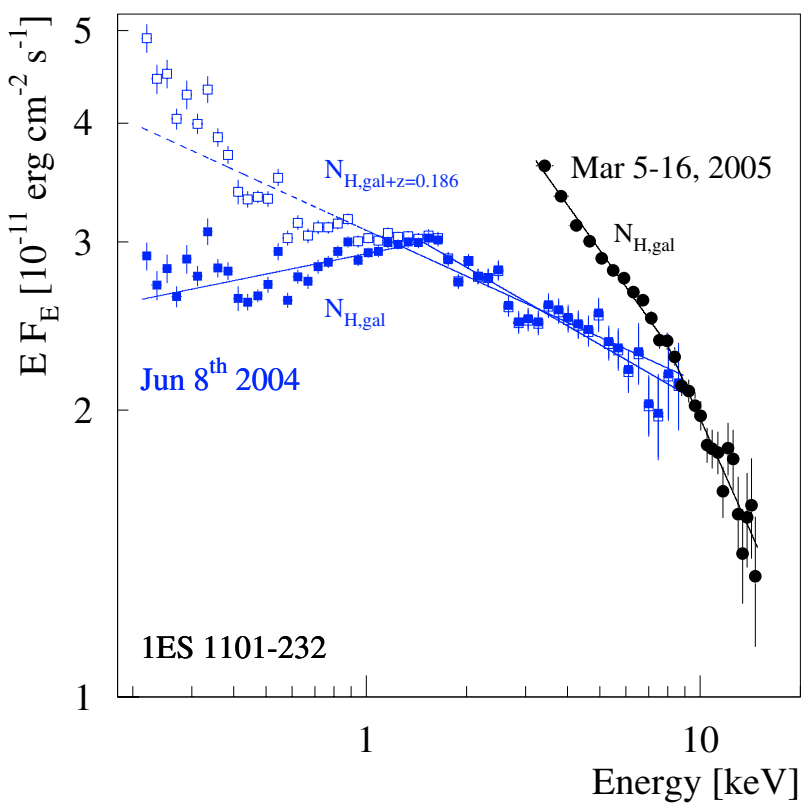

Fig. 5. Unfolded X-ray spectra from $1 \mathrm{ES} 1101-232$ in $E F(E)$ representation. Points between 3-15 keV labeled Mar. 5-16, 2005 are from RXTE, the line shows a broken power-law fit to the data. Points labeled Jun. 8th, 2004 between $0.2-10 \mathrm{keV}$ are from XMM-Newton. The filled squares represent the reconstructed spectrum under the assumption of pure Galactic hydrogen absorption, and can be fit by a broken power law. For the spectrum shown with open squares, in addition to Galactic also absorption in the source was allowed, under the assumption of a pure power-law emission spectrum.

\subsubsection{Optical data}

During the March 2005 campaign, optical data on 1ES 1101-232 were obtained using the ROTSE $3 \mathrm{c}$ telescope (Akerlof et al. 2003), which is located at the HESS site. The ROTSE 3c telescope is a fast slewing robotic telescope with a $45 \mathrm{~cm}$ mirror and a wide field of view of $1.85^{\circ}$. To collect as much light as possible ${ }^{4}$, no optical filter is used; the ROTSE passband is 400 to $900 \mathrm{~nm}$.

During each of the 11 nights, typically 18 frames of $60 \mathrm{~s} \mathrm{ex-}$ posure time were obtained. After quality selection, 141 frames were used for analysis. The standard ROTSE calibration chain ${ }^{5}$ delivered bias-corrected, flatfielded images. To obtain object magnitudes, the standard ROTSE procedure involves a vignetting correction and a relative photometry to a large number of comparison objects in the field of view, using their USNO-A2 (Monet et al. 1998) $R$-band magnitudes as reference. The standard ROTSE photometry however failed to analyze the data from 1ES 1101-232 because of source confusion, therefore a manual photometry was performed, as described in the following.

Three temporally-stable comparison stars close to 1ES 1101-232 were selected. Two of these stars have been identified by Smith et al. (1991) as calibrators and have known photometric multi-color data, the third star was used for additional cross-checks. An aperture of $R=7.5^{\prime \prime}$ was used for photometry. The stability of our photometry procedure was verified with several individual frames, by checking the correlation between measured fluxes and $C$-band magnitudes of the reference and various other field stars. $C$-band magnitudes

\footnotetext{
${ }^{4}$ The main purpose of the ROTSE telescopes are optical afterglow observations of gamma ray bursts.

${ }^{5}$ http://rotse1.physics.1sa.umich.edu/equipment/
}

(579-642 $\mathrm{nm}$ bandpass) were derived from the CCD astrograph catalog UCAC2 (Zacharias et al. 2004).

To obtain a light curve and check for variability of the optical flux, for each frame an effective $C$-band magnitude of 1ES 1101-232 was derived, using the three comparison stars as calibrators. The optical light curve so derived exhibited only marginal flux variations, nightly flux averages showed changes below 0.1 mag.

To derive an average optical flux of the BL Lac for use in the SED, an absolute flux calibration and subtraction of the host galaxy flux had to be performed. However, two facts caused this to be very difficult: the wide ROTSE bandpass, and the measured flux of $m_{C}=16.03 \mathrm{mag}$ which is close to the flux from the host galaxy $\left(m_{R}=16.41 \mathrm{mag}\right)$. Therefore, only an upper limit and a tentative lower limit of the optical flux could be derived.

In order to subtract the flux from the host galaxy, it was verified that the spectra of the two comparison stars used (Smith et al. 1991) are similar to template spectra of elliptical hosts (Fukugita et al. 1995) at the redshift of 1ES 1101-232. Applying the de Vaucouleurs profile with an effective radius of the host galaxy of $R_{\mathrm{e}}=4.1^{\prime \prime}$ and its total flux of $m_{R}=16.41 \mathrm{mag}$ as measured by Falomo \& Ulrich (2000), we concluded that $40 \%$ of the measured intensity comes from the host galaxy. If the BL Lac had also a similar spectrum, the apparent magnitude of the BL Lac would then be $m_{R}=16.4$ mag.

However, the wide bandpass of the ROTSE instrument causes this estimate to be quite susceptible to the actual BL Lac spectrum, which is expected to be much harder than the spectrum of the host galaxy. To estimate the magnitude of this effect, power-law spectra $S_{v} \propto v^{-\alpha}$ with $\alpha$ between 1.0 and -0.5 were simulated, under the assumption of a flat or symmetric response of the ROTSE detector between $400 \mathrm{~nm}$ and $900 \mathrm{~nm}$, and correction factors between 1.20 and 1.44 to the $R$-band flux were derived.

Magnitudes were finally corrected for Galactic extinction, using a $B$-band extinction $A_{B}=0.254$ mag (provided by NED; from Schlegel et al. (1998)) and following the extinction laws of Cardelli et al. (1989). Fluxes were derived from the magnitudes using the absolute calibrations by Bessel (1979). With this, an upper limit (assuming $\alpha=-0.5$ ) of $S_{R}=2.6 \mathrm{mJy}$ was derived, and a tentative lower limit (using no correction factor) of $S_{R}=1.0 \mathrm{mJy}$.

\subsection{Observations in June 2004}

The XMM-Newton observations on 1ES 1101-232 were conducted on June 8, 2004, as part of the pointings for XMM-Newton proposal 20592. A continuous pointing of $19.6 \mathrm{ks}$ was scheduled such that HESS observations could be conducted simultaneously. Simultaneous ROTSE 3c observations could not be performed because the telescope was under repair during that period.

\subsubsection{X-ray data}

The XMM-Newton data files were processed with xmmsas 6.5 .0 following standard procedures as described in the SAS Handbook and calibration documents (Kirsch et al. 2006), where also systematic errors are discussed. For our analysis, the most recent calibration files as available in January 2006 were used. Spectral and timing analysis was performed with XSPEC 11.3.1d and XRONOS 5.21, respectively. 
The EPIC instruments during this pointing were set in timing (PN and MOS 1 cameras) and small window (MOS 2 camera) mode, since one of the goals was to study spectral variability at the shortest possible timescales without pile-up problems, especially if the source were found in a very bright state.

During this observation, however, the source was characterized by a constant flux on all timescales. Analysis of the light curves extracted in different energy bands, and the corresponding hardness ratios, showed no indication of spectral variability as well. Therefore the entire dataset was used to derive a time-integrated energy spectrum. In the following, since the MOS 1 data are in agreement with the other instruments but present a higher noise, the analysis was restricted to $\mathrm{PN}$ and MOS 2

Pile-up effects in the PN and MOS 2 data were checked with epatplot, and a mild pile-up was found for MOS 2, that can be removed considering single pixel events only (PATTERN $=0$ ), which were compared to spectra extracted with patterns $0-12$ from different annular source regions (Molendi \& Sembay 2003). For the PN, since in timing mode, PATTERN $\leq 4$ was selected (single and double pixel events). For both instruments, quality $\mathrm{FLAG}=0$ was used. The total livetime after screening for soft proton flares was $17.005 \mathrm{ks}$ for $\mathrm{PN}$ and $16.653 \mathrm{ks}$ for MOS 2.

For MOS 2, source counts were extracted from a circle with radius $45^{\prime \prime}$ centered on the source centroid (obtained through eregionanalyse). The background was extracted from the nearest source-free region on the peripheral CCDs, with radius $90^{\prime \prime}$. A check using different locations on the peripheral CCDs, and the same source position on the blank-sky fields (available at the XMM-Newton Science Operating Center), showed no relevant differences, as was expected given the source rate $\left(\sim 8 \mathrm{cts} \mathrm{s}^{-1}\right)$ and spectrum. For the $\mathrm{PN}$ (which in timing mode has only one-dimensional imaging capabilities), photons were selected from rows $27 \leq$ RAWX $\leq 47$ (i.e., \pm 10 RAW pixels around the source strip), and $2 \leq$ RAWX $\leq 18$ for the background. To avoid the increased noise at low energies, the energy range for $\mathrm{PN}$ was restricted to $0.5-10 \mathrm{keV}$, while events down to $0.2 \mathrm{keV}$ were accepted for MOS 2, as allowed by the latest calibration (Kirsch et al. 2006). Spectra were rebinned with grppha to have at least 20 counts per channel. Response matrices and ancilliary files were produced with rmfgen and arfgen.

The spectra were fitted with source models plus interstellar medium absorption, using the model phabs with abundances from Wilms et al. (2000). The absorbing column density was fixed to the Galactic value $N_{\mathrm{H}}=5.76 \times 10^{20} \mathrm{~cm}^{-2}$, but also a model with additional free absorption at the source $(z=0.186)$ was tested. The host galaxy of 1ES 1101-232 is not a normal elliptical galaxy, the measured boxy isophotes (Falomo \& Ulrich 2000) may be indicative of extra absorbers, though in previous $\mathrm{X}$-ray observations especially with BeppoSAX such possibility was statistically disfavoured (see Sect. 4.3).

The PN and MOS 2 spectra were inspected separately, but finally the data from the two instruments were fitted together, with a free constant to allow for a different normalization between the two instruments (which remained within 3\%). The results from the combined fit are reported in Table 5. The $\chi^{2}$-values of the combined fits are relatively high, but to a large extent this is owing to residual cross-calibration uncertainties (though strongly improved with SAS 6.5) combined with large statistics.

With Galactic absorption, a single power law does not provide an acceptable fit for the single detectors as well as for the combined fit $\left(\chi_{\text {red }}^{2}=1.880\right.$ for 1547 d.o.f.). A broken power-law model significantly improves all fits, with $\chi_{\text {red }}^{2}=$ 1.195 (1545 d.o.f., null-hypothesis probability $2 \times 10^{-7}$ ) for the combined fit. The hard $\left(\Gamma_{1}=1.94 \pm 0.01\right)$ and soft $\left(\Gamma_{2}=2.19 \pm\right.$ $0.01)$ spectral indices locate the X-ray peak of the SED at the break energy $E_{\text {break }}=1.1 \mathrm{keV}$. Looking into the individual camera fits, the soft slopes are in agreement $\left(\Gamma_{2, \mathrm{PN}}=2.19_{-0.02}^{+0.01} \mathrm{vs}\right.$. $\Gamma_{2, \operatorname{MOS} 2}=2.21_{-0.02}^{+0.01}$, while break positions and hard slopes are slightly differing $\left(\Gamma_{1, \mathrm{PN}}=1.98_{-0.02}^{+0.01}, E_{\text {break }, \mathrm{PN}}=1.09_{-0.02}^{+0.01} \mathrm{keV}\right.$, vs. $\left.\Gamma_{1, \operatorname{MOS} 2}=1.91_{-0.02}^{+0.01}, E_{\text {break,MOS } 2}=1.45_{-0.02}^{+0.01} \mathrm{keV}\right)$; the respective fits are well acceptable for PN but slightly disfavoured for MOS 2 (null-hypothesis probability: PN 5\%, MOS $20.3 \%$ ). Reasons for the discrepancies are the different fit ranges towards the soft end of the spectra, and the already mentioned calibration uncertainties.

The combined broken power-law fit, considering $N_{\mathrm{H}}$ as a free parameter, yields a column density of $N_{\mathrm{H}}=5.8 \pm 0.7 \times$ $10^{20} \mathrm{~cm}^{-2}$ which is in very good agreement with the Galactic value. To test whether additional absorption at the source is compatible with the data, a single power law model with Galactic absorption and free absorption at $z=0.186$ was investigated. The results are less favoured than the fits with pure Galactic absorption (null-hypothesis probability: PN: 0.7\%, MOS 2: $10^{-15}$, combined fit: $3 \times 10^{-11}$, extra absorption $N_{\mathrm{H}} \sim 3 \times 10^{20} \mathrm{~cm}^{-2}$ ), and provide more skewed residuals with an excess at lower energies less compatible with the hypothesis of higher absorption. The difference to the models with Galactic absorption is however not large, also considering the fact that the MOS 2 spectrum still exhibits some unmodeled residua at low energies $(0.4-0.5 \mathrm{keV})$, which could possibly be because of the mentioned detector calibration uncertainties and/or imperfect modeling of the hydrogen absorption (even free abundances improve only slightly the residuals).

To obtain the unfolded energy spectrum for the SED, the results of the combined fit were used. The residuals of the MOS 2 data to the respective MOS 2 model were multiplied with the model derived for the PN. With this prescription, the absolute flux calibration from PN is trusted, while the full spectral energy range from MOS 2 can be used. Finally, the data were rebinned logarithmically in energy. The unfolded spectrum is shown in Fig. 5, together with a similarly derived spectrum under the assumption of a pure power law and additional absorption at the source, for comparison. The model fits in Fig. 5 were derived from the unfolded spectra and are shown to indicate the size of the residuals. We note that the unfolded spectra do not strongly depend on the exact shape of the modeled spectrum that is used in the unfolding procedure (using for example a pure power law instead of a broken power law yields a compatible spectrum within errors).

To conclude, while small amounts of extra absorption at the source - which would soften the slope below $1 \mathrm{keV}$ - cannot be excluded based on the XMM-Newton spectra alone, there is good evidence that the X-ray spectrum from 1ES 1101-232 exhibited a peak in the SED at $\sim 1 \mathrm{keV}$ during our observations, similar to earlier X-ray observations of this source.

For the June 2004 SED discussed in the next section, the data of the entire HESS June data set (i.e., \pm 3 days from the XMM-Newton pointing) were taken, in order to obtain a significant signal from the VHE data. Quantitatively, 3.4 (66\%) of the $5.1 \mathrm{~h}$ (MOS 2) XMM-Newton observations have simultaneous HESS data. These $3.4 \mathrm{~h}$ conversely represent $37 \%$ of the June 2004 HESS data set $(9.1 \mathrm{~h}$ of on-source observation time). 
Table 4. Observation log of the two X-ray satellite observations on 1ES 1101-232 used for this paper.

\begin{tabular}{lllll}
\hline \hline Satellite & Observation ID & Observation dates & Pointings & Used detectors and live time \\
\hline RXTE & 91123 & March 5-16, 2005 & 48 & PCU 2: 89.60 ks \\
XMM-Newton & 205920601 & June 8, 2004 & 1 & MOS 2 (thin filter): 17.65 ks \\
& & & & PN (thin filter): 17.01 ks \\
& & & OM: $V$ 1.60, B 1.88, U 1.60, UVW1 4.00, UVM2 4.00 ks \\
\hline
\end{tabular}

\subsubsection{Optical data}

During the observations on 1ES 1101-232, the optical monitor (OM) onboard XMM-Newton took five exposures with five different filters, from the $V$ to the $U V$ band, with a window in fast mode on our target. Details are given in Table 4. As in the EPIC instruments, no variability was found in any OM exposure. Therefore, a photometric spectrum was extracted from all filters.

$\mathrm{OM}$ data were processed with xmmsas 6.5.0. Only data from the imaging mode were used in the following. OM count rates were extracted using the point source analysis procedure of the OM photometry program. An aperture of $R=6^{\prime \prime}$ was used for the source, background counts were extracted from an annulus with $7^{\prime \prime}<R<12.5^{\prime \prime}$, for all filters. Count rates were converted into fluxes according to the prescriptions of the XMM-Newton watch-out pages ${ }^{6}$. The conversion factors for a white dwarf were used, as recommended by the OM calibration scientist (Nora Loiseau, priv. comm.).

The point spread function of the $\mathrm{OM}$ is considerably wider in the UV ( $U V W 1$ and $U V M 2$ filters) than in the other filters, requiring specific aperture corrections in those bands. The default aperture for these filters could not be used because of bright sources in the corresponding background annulus, therefore the same source and background apertures were used as for the other filters. The validity of the aperture correction was tested by selecting different source and background aperture sizes. The systematic error on fluxes derived with the UV filters was estimated to be $\sim 20 \%$.

In the $V$ and $B$ bands, a contribution from the host galaxy needs to be corrected. Using the same de Vaucouleurs profile as in Sect. 4.1.2, $61 \%$ and $15 \%$ of the host galaxy flux were estimated to fall into the source and background apertures, respectively. These fractions of the host galaxy flux were subtracted, using its $R$-band magnitude $m_{\mathrm{R}}=16.41 \mathrm{mag}$ from Falomo \& Ulrich (2000) and the elliptical galaxy spectral template from Fukugita et al. (1995).

Fluxes were finally corrected for Galactic extinction, again using $A_{B}=0.254 \mathrm{mag}$. The derived optical spectrum is shown in the SED in Fig. 6, lower left panel. Error bars at the two UV flux points are from our systematic uncertainty estimate. We note that the U-filter flux is probably the most reliable flux estimate for the BL Lac, because of the mentioned uncertainties in the UV filters and because of the host galaxy influence at larger wavelengths.

\subsection{Previous $X$-ray observations of 1ES 1101-232}

1ES 1101-232 has already been observed in previous pointed X-ray observations with ROSAT (Wolter et al. 1998), BeppoSAX (Wolter et al. 2000) and XMM-Newton (Perlman et al. 2005). Spectra and fluxes were significantly different comparing individual observations, but the flux changes were not large $( \pm 25 \%)$. For example, the two spectra taken with BeppoSAX in 1997 and 1998 (dubbed as high and low state in Wolter et al. 2000) only showed a significant change above the

\footnotetext{
${ }^{6}$ http://xmm.vilspa.esa.es/sas/new/watchout
}

peak at $\sim 1.4 \mathrm{keV}$, with a total flux difference of $50 \%$. Results from the different observations, including the two observations discussed in this paper, are summarized in Table 5.

In all observations, pure intrinsic power laws were rejected if only Galactic absorption values were assumed. All observations could be modeled with a pure power law, under the assumption of extra absorption of the order of $N_{\mathrm{H}} \simeq 3 \times 10^{20} \mathrm{~cm}^{-2}$. Nevertheless, in cases where the statistical accuracy was sufficient (SAX I, SAX II, XMM II, cf. Table 5), significantly better fits were derived, using only Galactic absorption and a curved intrinsic spectrum (usually modeled by a broken power law).

Assuming hence no extra absorption at the source, the lowenergy peak in the SED of 1ES 1101-232 has in most observations been located in the $\sim 0.5-3.5 \mathrm{keV}$ range. From the difference of the position of the spectral break derived from the data set SAX I (see Table 5) by Wolter et al. (2000) on the one hand and by Donato et al. (2005) on the other hand, and also following the discussion in Perlman et al. (2005), we conclude that a broken power law is only an approximation of the real spectrum. Nevertheless, this does not affect the SED modeling presented in the next section, as the used unfolded XMM-Newton X-ray spectrum does not significantly change if the broken power law model is replaced by a curved model, such as the one described in Fossati et al. (2000).

\section{Discussion}

\subsection{Constraints on the extragalactic background light}

The detection of VHE $\gamma$-ray emission from 1ES 1101-232 was used to constrain the density of the EBL flux in the wavelength range of $\sim 0.75-3.5 \mu \mathrm{m}$ (AHA06a and Sect. 3.8). The measured average photon index of $\Gamma=2.9$ either precludes high EBL levels, or indicates a much harder instrinsic spectrum than seen or expected in other VHE blazars. For the purpose of this paper, the idea was adopted that the intrinsic VHE $\gamma$-ray spectrum of $1 \mathrm{ES} 1101-232$ is restricted to $\Gamma_{\mathrm{VHE}, \mathrm{deabs}} \geq 1.5$. This limit is empirically derived in all blazars of lower redshift (where EBL corrections are less severe). It is also theoretically expected in present standard leptonic or hadronic scenarios for blazar emission employing shock acceleration models (Malkov \& Drury 2001), because the hardest energy index obtained for accelerated particles is $p=1.5$ which results in $\Gamma \geq 1.5$ for all emission processes which can realistically be assumed (AHA06a). As shown in AHA06a, the limit of $\Gamma_{\mathrm{VHE}, \text { deabs }} \geq 1.5$ applied to the measured spectrum of 1ES 1101-232 results in an EBL upper limit of $v F_{v}=14 \mathrm{nWm}^{-2} \mathrm{sr}^{-1}$ at $1.5 \mu \mathrm{m}$ for typical EBL shapes.

We note that harder photon spectra result if the low energy end of the particle distribution is altered from the assumptions mentioned above. A variety of possibilities have been discussed in the literature. Relativistic Maxwellian (pileup) particle energy spectra, produced in a balance of stochastic acceleration and radiative cooling (Schlickeiser 1985; Henri \& Pelletier 1991), have previously been invoked in the context of blazar spectra (Saugé $\&$ Henri 2004). Radiative cooling of an injection spectrum flatter than $\gamma^{-2}$ could also result in a pileup spectrum (Pinkau 1980; 
Table 5. Spectral fit results of selected X-ray observations on 1ES 1101-232.

\begin{tabular}{|c|c|c|c|c|c|c|c|c|}
\hline Obs. & $\begin{array}{l}F_{2-10 \mathrm{keV}} \times \\
10^{-11} \mathrm{erg} \mathrm{cm}^{-2} \mathrm{~s}^{-1}\end{array}$ & 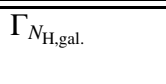 & 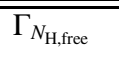 & $\begin{array}{l}N_{\mathrm{H}} \times \\
10^{20} \mathrm{~cm}^{-2}\end{array}$ & 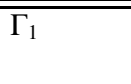 & $\begin{array}{l}E_{\text {break }} \\
\text { keV }\end{array}$ & 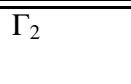 & Reference \\
\hline ROSAT & $2.33_{-0.27}^{+0.30}$ & $2.23_{-0.03}^{+0.04}$ (a) & $2.43_{-0.08}^{+0.08}$ & $6.8_{-0.4}^{+0.3}$ & $2.07_{-0.22}^{+0.09}$ & $0.69_{-0.25}^{+0.24}$ & $2.42_{-0.09}^{+0.12}$ & (1) \\
\hline SAX I & $3.76^{-0.21}$ & $1.97_{-0.02}^{-0.03}(\mathrm{~b})$ & $2.03_{-0.04}^{+0.05}$ & $8.9_{-2.7}^{+3.4}$ & $1.59_{-0.14}^{+0.15}$ & $1.36_{-0.25}^{+0.29}$ & $2.05_{-0.04}^{+0.03}$ & (2) \\
\hline SAX I ${ }^{\dagger}$ & 3.68 & $-\quad-0$ & $2.01_{-0.06}^{-0.04}$ & $8.5_{-2.0}^{+3.2} \dagger \dagger$ & $1.73_{-0.09}^{+0.14}$ & $3.45_{-0.52}^{+0.25}$ & $2.19_{-0.11}^{-0.04}$ & (3) \\
\hline SAX II & 2.55 & $2.19_{-0.03}^{+0.03}(\mathrm{c})$ & $2.25_{-0.03}^{+0.00}$ & $8.3_{-1.2}^{+2.1}$ & $1.80_{-0.22}^{+0.16}$ & $1.34_{-0.26}^{+0.42}$ & $2.29_{-0.04}^{+0.04}$ & (2) \\
\hline XMM I & 2.3 & $2.23_{-0.01}^{+0.02}(\mathrm{~d})$ & $2.40_{-0.03}^{+0.03}$ & $10.9_{-0.6}^{-1.2}$ & $* 2.06_{-0.12}^{+0.12}$ & - & ${ }^{*} 2.38_{-0.15}^{+0.04}$ & (4) \\
\hline XMM II & $3.74_{-0.02}^{+0.01}$ & $2.11_{-0.01}^{+0.01}(\mathrm{e})$ & $2.19_{-0.01}^{+0.01}$ & $8.53_{-0.17}^{+0.17}$ & $1.94_{-0.01}^{+0.01}$ & $1.11_{-0.04}^{+0.05}$ & $2.19_{-0.01}^{+0.01}$ & this work \\
\hline XTE & $5.07 \pm 0.02$ & - & - & - & $2.49_{-0.02}^{+0.02}$ & $7.9_{-0.9}^{+1.0}$ & $2.78_{-0.11}^{+0.16}$ & this work \\
\hline
\end{tabular}

(a-e): Values from spectral fits with low probablility: (a): $0.7 \%$. (b): $<2 \%$. (c): $<0.5 \%$. (d): $<10^{-9}$ (e): $\ll 10^{-10}$. ${ }^{\dagger}$ : Reanalysis of the data set "SAX I" presented originally by Wolter et al. (2000). ${ }^{\dagger \dagger}$ : Donato et al. (2005) used $N_{\mathrm{H}, \mathrm{gal} .}=6.05 \times 10^{20} \mathrm{~cm}^{-2}$ and additional absorption at the source $\left(2.4_{-2.0}^{+3.2} \times 10^{20} \mathrm{~cm}^{-2}\right)$; for simplicity, the sum of these two values is quoted here. $\Gamma_{1}, E_{\text {break }}, \Gamma_{2}$ from a broken power-law fit, except ${ }^{*}: \Gamma_{1}=$ $\Gamma_{0.5-1.0 \mathrm{kev}}, \Gamma_{2}=\Gamma_{4.0-10.0 \mathrm{keV}}$. The XMM II values are from the combined PN/MOS 2 fits as described in the text, the flux normalisation is from PN only. The XTE row is detached to emphasize that the data are not sensitive to the break seen by all other instruments in the $\sim 0.5-3.5 \mathrm{keV}$ range. References: (1) Wolter et al. (1998); (2) Wolter et al. (2000); (3) Donato et al. (2005); (4) Perlman et al. (2005).

Kardashev 1962). Also bulk motion Comptonization of ambient low-frequency photons by a cold unshocked ultrarelativistic jet with a very large Lorentz factor of the order of $10^{6} . .10^{7}$ could yield a narrow, line-like spectrum (Aharonian et al. 2002a).

Katarzyński et al. (2006) used the total VHE $\gamma$-spectrum of 1ES 1101-232 from AHA06a, together with archival X-ray data that were not taken simultaneously with the HESS data, and showed that emission from a power-law type particle spectrum with a low energy cut-off at large Lorentz factors $\sim 10^{5}$ can produce an intrinsic VHE $\gamma$-spectrum as hard as $\propto v^{+\frac{1}{3}}$. This would allow for an EBL level of $v F_{v}(1.5 \mu \mathrm{m}) \simeq 20 \mathrm{nWm}^{-2} \mathrm{sr}^{-1}$ and fit the VHE data. We note that our simultaneous data show no indication for such effects in the synchrotron branch. For the purpose of this paper, we refrain from discussing such spectra further.

\subsection{Spectral energy distribution of 1ES 1101-232}

For the construction of the SED, the deabsorbed data were taken, using what we consider the best available deabsorption prescription. The optical and X-ray data were corrected for Galactic absorption, see Sects. 4.1.2, 4.2.1, and 4.2.2. The VHE $\gamma$-ray spectra were deabsorbed, using two possible levels of the present day EBL photon field: the maximum value of $v F_{v}=14 \mathrm{nWm}^{-2} \mathrm{sr}^{-1}$ at $1.5 \mu \mathrm{m}$, and a minimum value of $10 \mathrm{nWm}^{-2} \mathrm{sr}^{-1}$ corresponding to the lower limit placed by Galaxy counts (Madau \& Pozzetti 2000). We note that the galaxy counts are presumably below the actual EBL density because of missed light (Totani et al. 2001). To derive the optical depth for the VHE $\gamma$-rays, the phenomenological EBL curve as used in AHA06a was applied, after scaling to match the given maximum and minimum EBL densities and to take galaxy evolution effects into account, as explained in Sect. 3.6. A redshift correction of the frequencies shown in the SED (to account for the difference of apparent and restframe wavelength) was not performed, as the correction would be dominated by the uncertainty of the emitting region's Doppler factor.

In Fig. 6 the SEDs of 1ES 1101-232 are shown for the two periods for which broadband data together with the HESS VHE measurements have been obtained. The upper panel of Fig. 6 contains the average fluxes of the March 2005 campaign (i.e., March 5-16, 2005), as derived from HESS, RXTE, and ROTSE 3c data. As described in Sect. 4.1.1, the data have not been restricted to true simultaneity (i.e., on minutes timescale).
However, all data were taken during the same observing nights, with no significant (VHE) or only mild (X-ray, optical) variations between days, and there is no sign of variability in these data on timescales shorter than a day.

The SED shown in the lower panel of Fig. 6 has a lesser degree of simultaneity, because it contains the average VHE $\gamma$-ray spectrum obtained from the HESS June 2004 observations (i.e., June 5-10, 2004), together with the X-ray and optical data as derived from the XMM-Newton observation performed on June 8 (see Sect. 4.2.1).

\subsection{Model-independent considerations}

Independent of specific emission scenarios, the broadband data from 1ES 1101-232 presented here show some remarkable features:

VHE peak: the hard intrinsic VHE $\gamma$-ray spectrum $\left(\Gamma_{\text {deabs }} \lesssim\right.$ 2) requires that the VHE $\gamma$-ray flux peak is located at $>3 \mathrm{TeV}$ and that the measured VHE photons do come from below that peak, unless the lowest possible EBL level is assumed and errors on the VHE $\gamma$-ray spectrum are exploited to the limits. The VHE $\gamma$-ray spectrum does not show hints of curvature. A steepening towards higher energies would indicate the position of the SED high-energy peak at $\sim 3 \mathrm{TeV}$ (either due to a break in the particle distribution, or because of Klein Nishina effects), but the data are insufficient to claim such a steepening.

Comparison 2004/2005: the source was in a different state in March 2005 compared with the June 2004 period, judging from the X-ray data. While the X-ray flux and spectrum in June 2004 were comparable to previous X-ray measurements (see Table 5), the X-ray spectrum in March 2005 as measured with RXTE shows a higher flux and a fairly soft spectrum, softer than any previously measured X-ray spectrum from this source. The synchrotron peak (usually at $\sim 0.5-3.5 \mathrm{keV}$ ) cannot have shifted to higher energies during the March 2005 high state, the XTE spectrum precludes a peak above $\sim 3 \mathrm{keV}$. Despite the different X-ray flux states in March 2005 and June 2004, there is no evidence for a change of the VHE $\gamma$-ray spectrum between the two periods; nevertheless, statistical errors from the June 2004 data sets could allow for a factor of up to about two flux difference between the two periods.

$X$-ray variability: the X-ray light curve in March 2005 only exhibits mild variations. Under the assumption of $\gamma^{-2}$-type 


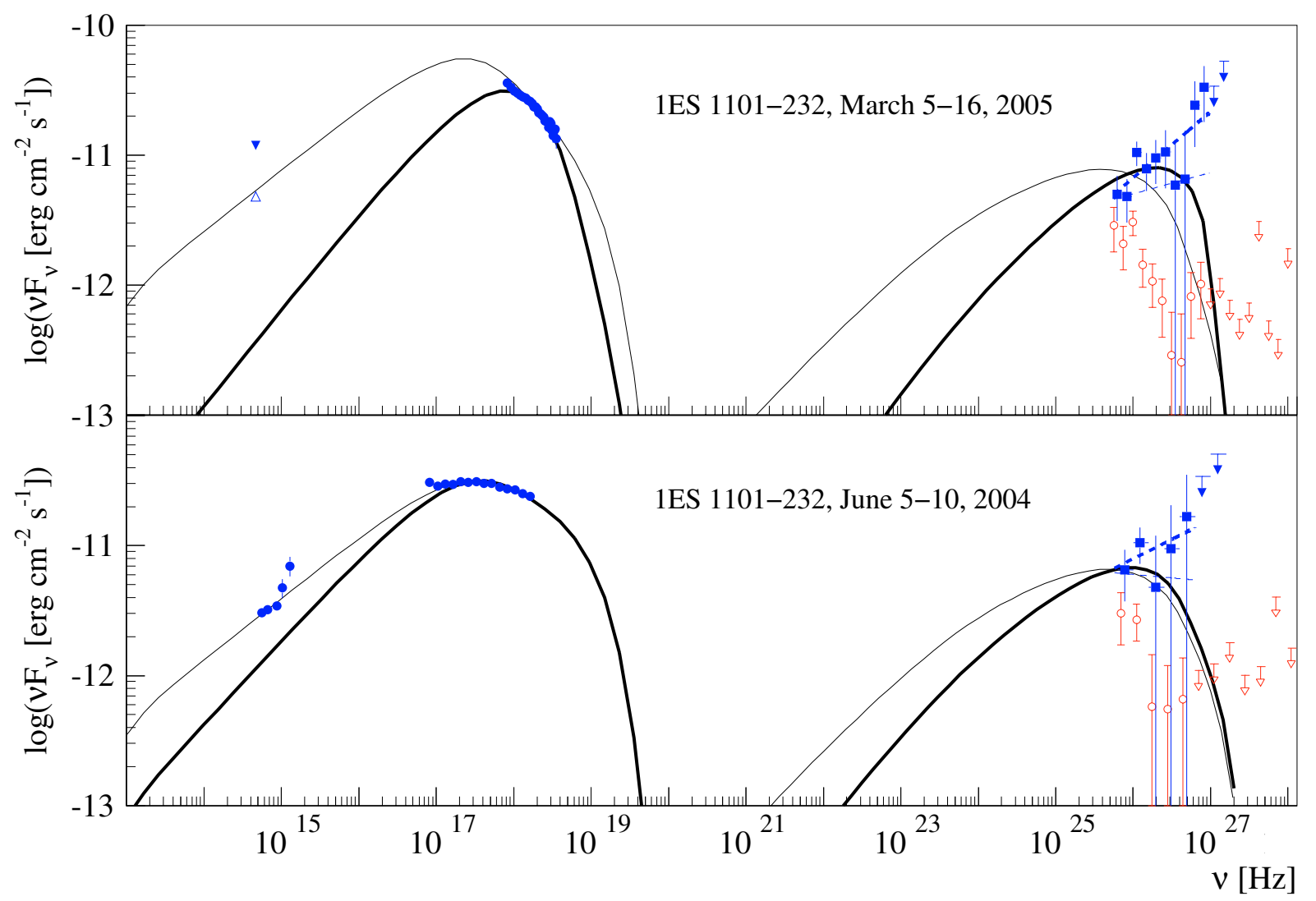

Fig. 6. Spectral energy distribution of 1ES 1101-232. Upper panel: data from March 5-16, 2005. X-ray data are from RXTE. In the optical band, an upper limit (filled triangle) and a tentative lower limit (open triangle) from ROTSE 3c data are shown, see Sect. 4.1.2 for details. In the VHE band, the measured HESS spectrum (red, open symbols) and the deabsorbed spectrum using a maximum EBL level of $14 \mathrm{nWm}^{-2} \mathrm{sr}^{-1}$ at $1.5 \mu \mathrm{m}$ (see text) are shown; for better visibility, all open symbols were slightly shifted to the left, to $90 \%$ of the respective original frequency. The thick dashed line is a power-law fit to the deabsorbed data as plotted, while the thin dashed line indicates the effect if the EBL is lowered to the minimum level of $10 \mathrm{nWm}^{-2} \mathrm{sr}^{-1}$. The latter value corresponds to the minimum level of the EBL as derived from known resolved galaxy counts. Thick and thin solid curves denote results from a single zone SSC model. The thick curves represent a model that was optimized to fit the HESS and X-ray data, while the thin lines denote a model with an electron distribution $N_{\gamma} \propto \gamma^{-2}$ below the break. Lower panel: data from June 2004. X-ray and optical data were derived from an XMM-Newton pointing on June 8, 2004. In the VHE band, HESS data taken between June 5-10, 2004, are shown, using the same procedure as described for the upper panel. Solid curves denote results from a single zone emission model, also following the same prescription as for the upper panel.

injection spectra, it seems unlikely that the soft RXTE spectrum from that period is governed by emission from a cooled particle spectrum. The spectrum could reflect the cut-off of the acceleration spectrum, but depending on the assumed scenario, other options like softer injection spectra (e.g., Ghisellini et al. 2002) are also possible.

Leptonic emission spectrum: one can compare X-ray and VHE $\gamma$-ray spectral indices under the assumption that electrons from the same energy band with $N_{\gamma} \propto \gamma^{-p}$ are emitting X-rays via synchrotron and VHE photons via IC processes. The rather flat X-ray spectrum over nearly two decades in energy, as seen with XMM-Newton in 2004, constrains $p$ to $2.9<p<3.4$, and therefore requires that the VHE $\gamma$-ray emission is in the Thompson regime $\left(1.9<\Gamma_{\mathrm{IC}}<2.2\right)$ to be still in agreement with the measured VHE $\gamma$-ray spectrum $\left(\Gamma_{\text {Jun. } 2004}=1.70 \pm 0.47\right)$. Assuming that the VHE $\gamma$-ray spectrum was constant throughout the observation periods $\left(\Gamma_{\text {total }}=1.51 \pm 0.17\right)$ would require either to drop the initial assumption about the common spectral range of the emitting electrons, or to lower the EBL to the lowest possible value of $v F_{v}(1.5 \mu \mathrm{m})=10 \mathrm{nWm}^{-2} \mathrm{sr}^{-1}$. For the March 2005 data set, a common energy band of the electron spectrum cannot account for the X-ray and VHE $\gamma$-ray emission.
Intrinsic source luminosity: depending on the assumed emission mechanism and EBL density, estimates of the intrinsic luminosity $L_{\mathrm{VHE}}$ of the VHE $\gamma$-ray emitting region in 1ES 1101-232 can reach unusually large values. Following Sikora et al. (1997), the intrinsic luminosity would be $L_{\mathrm{VHE}}>10^{39} \mathrm{erg} \mathrm{s}^{-1}$ under the assumption of SSC VHE $\gamma$-ray emission from a moving blob with Doppler factor $\delta \sim 30$. Since however the emission seems constant on timescales of days or maybe even years, a stationary jet model could also be invoked, leading to estimates of $L_{\mathrm{VHE}}>$ $10^{42} \mathrm{erg} \mathrm{s}^{-1}$. Adopting like in Katarzyński et al. (2006) the "best fit" EBL model from Kneiske et al. (2004), with an EBL density approaching $v F_{v}(1.5 \mu \mathrm{m}) \sim 20 \mathrm{nWm}^{-2} \mathrm{sr}^{-1}$, would increase the intrinsic luminosity estimates to $>10^{40} \mathrm{erg} \mathrm{s}^{-1}$ for the moving blob and even $>10^{43} \mathrm{erg} \mathrm{s}^{-1}$ for the stationary scenario.

\subsection{One-zone leptonic emission model}

Neither flux correlations between the X-ray and VHE bands nor fast variability (i.e., on sub-day or even sub-hour timescale) could be established with the present data set. Therefore, there is no clear indication that would favour one-zone emission models, either leptonic or hadronic. Past experience (e.g., Aharonian et al. 2005a) has shown that both hadronic and leptonic scenarios 
have enough free parameters to generally be able to explain the broad-band emission from blazars, if no further arguments from variability can be invoked. Nevertheless, the reconstruced hard VHE $\gamma$-ray spectrum from 1ES 1101-232 challenges one-zone emission models.

A simple leptonic emission model was used, a timeindependent SSC model as described by Katarzyński et al. (2001), in an attempt to describe the measured VHE, X-ray, and optical data. The description of a one-zone homogeneous, spherical emitting region $R$ and a homogeneous magnetic field $B$ was adopted, which propagates with Doppler factor $\delta$ towards the observer. The high-energy electron distribution is modeled with a broken power law between Lorentz factors $\gamma_{\min }$ and $\gamma_{\max }$ with a break at $\gamma_{\mathrm{b}}$, and a density normalisation $K$. The two epochs have been considered independently. In the March 2005 data set, the most stringent constraint on the SED comes from the hard $\gamma$-ray spectrum extending towards $3 \mathrm{TeV}$. In the June 2004 case, the $\gamma$-ray part of the spectrum is easier to reproduce because of the smaller number of events and the slightly softer spectrum, while the X-rays are more constraining with a rather flat spectrum over a large frequency range.

The results from two parameter sets for each SED are shown in Fig. 6 and Table 6. In case I, $p_{1}$ (the energy index of the particles between $\gamma_{\min }$ and $\gamma_{\mathrm{b}}$ ) was set to 2 , as expected from an uncooled, shock-accelerated particle distribution. With $\gamma_{\mathrm{b}, 2005}=1.8 \times 10^{5}$ and $p_{2,2005}=4.3\left(p_{2}\right.$ being the energy index between $\gamma_{\mathrm{b}}$ and $\gamma_{\max }$ ) for the March 2005 SED, and $\gamma_{\mathrm{b}, 2004}=1.8 \times 10^{5}, p_{2,2004}=3.6$ for the June 2004 data, good fits were obtained to the X-ray and optical data, respectively. As expected, the hard VHE spectra are not well reproduced when using $p=2$. The 2004 VHE data are still satisfactorily matched, but the very hard 2005 VHE $\gamma$-ray spectrum (reconstructed with the EBL density $14 \mathrm{nWm}^{-2} \mathrm{sr}^{-1}$ ), having also smaller statistical errors, is not well reproduced by the model. A simple $\chi^{2}$ test only yields a just over $2 \sigma$ compatibility between the data and the model. A lower EBL level improves the agreement with the data.

In case II, $p_{1}$ was chosen to be of the order of 1.5 , which can be expected for instance from particle acceleration at strong shocks in a relativistic gas. Better fits are then obtained for the $\gamma$-ray spectra at both epochs as illustrated in Fig. 6, especially for 2005 where the $\chi^{2}$ test now yields a $1 \sigma$ compatibility. In this scenario, the optical flux cannot be described within the SSC one-zone model, and has to be attributed to another component. This additional low frequency emission could come for example from some extended jet emission. Such an additional component is in any case needed to explain the emission at radio frequencies, which was measured with the Nancay radio telescope at $2.685 \mathrm{GHz}$ (see Aharonian et al. 2005a, for a description of the instrument and data processing). The flux levels obtained in June $2004(0.11 \pm 0.02 \mathrm{Jy}$, not simultaneous to the other data presented in this paper) and in March 2005 (0.08 $\pm 0.01 \mathrm{Jy}$, simultaneous to the data presented in this paper) were comparable to previous measurements (Griffith et al. 1994; Douglas et al. 1996).

To conclude, SSC one-zone models are globally able to reproduce the SED of 1ES 1101-232 from the X-rays to VHE $\gamma$-rays for the data set analyzed here. For the 2005 data set, an inclusion of the optical lower limit, obtained through the ROTSE 3c detector, is however statistically disfavoured. Moreover we should stress that with 1ES 1101-232 the limit of the capabilities of SSC one-zone models is reached, as also discussed in AHA06a. It is very difficult to get good fits for the shape of the VHE $\gamma$-ray tail of the observed spectra, as
Table 6. Physical parameters of the SSC one-zone model spectra. 2005 I and 2004 I, i.e. the scenarios with $p_{1}=2$, correspond to the models shown as thin solid lines in Fig. 6. 2005 II and 2004 II correspond to the models shown as thick solid lines. Doppler factor, magnetic field, $\gamma_{\min }$, and $\gamma_{\max }$ are in all four cases identical $\left(H_{0}=75 \mathrm{~km} \mathrm{~s}^{-1} \mathrm{Mpc}^{-1}\right)$.

\begin{tabular}{l|l|l|l|l}
\hline \hline & $2005 \mathrm{I}$ & $2005 \mathrm{II}$ & $2004 \mathrm{I}$ & $2004 \mathrm{II}$ \\
\hline$p_{1}$ & 2 & 1.5 & 2 & 1.7 \\
$p_{2}$ & 4.3 & 7.0 & 3.6 & 3.6 \\
$\gamma_{\mathrm{b}}$ & $1.8 \times 10^{5}$ & $3.8 \times 10^{5}$ & $1.8 \times 10^{5}$ & $1.8 \times 10^{5}$ \\
$K\left[\mathrm{~cm}^{-3}\right]$ & $3.5 \times 10^{2}$ & 15 & $9.0 \times 10^{2}$ & 40 \\
$R[\mathrm{~cm}]$ & $2.8 \times 10^{16}$ & $5.7 \times 10^{15}$ & $1.7 \times 10^{16}$ & $1.15 \times 10^{16}$ \\
\hline$\delta$ & \multicolumn{4}{|c}{25} \\
$B[\mathrm{G}]$ & \multicolumn{4}{|c}{0.1} \\
$\gamma_{\min }$ & \multicolumn{3}{|c}{$10^{3}$} \\
$\gamma_{\max }$ & \multicolumn{4}{|c}{$10^{6}$} \\
\hline
\end{tabular}

long as one keeps usual assumptions for particle acceleration mechanisms and does not assume an EBL level as low as $10 \mathrm{nWm}^{-2} \mathrm{sr}^{-1}$. The generated spectra deviate, especially for the March 2005 data, from the hard spectra obtained with HESS. Smaller statistical errors on the VHE $\gamma$-ray spectrum or an extension to higher energies (or both), further constraints on the size of the emitting zone from variability data, or a slight increase of the absorption by extragalactic background above the value of $14 \mathrm{nWm}^{-2} \mathrm{sr}^{-1}$, may reach the limit of one-zone SSC models. More complex (e.g., two-zone) scenarios may therefore be required.

\section{Conclusion}

Using the HESS Cherenkov telescope system, VHE $\gamma$-ray emission from 1ES 1101-232 was discovered. 1ES 1101-232 is currently the farthest object $(z=0.186)$ with confirmed redshift detected in VHE $\gamma$-rays. The VHE $\gamma$-ray spectrum favours a low level of the extragalactic background light at a few microns, close to the lower limit placed by galaxy counts.

The VHE data from 1ES 1101-232 were taken together with measurements in the X-ray and optical bands. The best SED from truly simultaneous observations could be constructed from a multifrequency campaign performed in March 5-16, 2005. The data revealed that the source was brighter in X-rays during this campaign than in any other previous X-ray observation, but did not show strong flaring activity in either the X-ray or the VHE band. The HESS data set is compatible with constant VHE $\gamma$-ray emission throughout all observation periods.

Using an EBL density of $v F_{v}=14 \mathrm{nWm}^{-2} \mathrm{sr}^{-1}$ at $1.5 \mu \mathrm{m}$ to deabsorb the VHE $\gamma$-ray spectrum, the HESS data result in a very hard intrinsic spectrum of $1 E S$ 1101-232, with a peak in the VHE power output above $3 \mathrm{TeV}$. The spectrum is harder $(\Gamma \simeq$ 1.5) than in parametrisations using SSC models. An EBL level below $14 \mathrm{nWm}^{-2} \mathrm{sr}^{-1}$ would soften the $\gamma$-ray spectrum nearly to $\Gamma \simeq 2.0$, which would ease the application of SSC scenarios but at the same time challenge EBL models. On the other hand, an EBL density above $14 \mathrm{nWm}^{-2} \mathrm{sr}^{-1}$ would result in an even harder $\gamma$-ray spectrum, therefore such high EBL levels can be excluded under the assumption that 1ES 1101-232 is similar to all VHE blazars known so far (AHA06a). A $\gamma$-ray spectrum in 1ES 1101-232 harder than $\Gamma \simeq 1.5$ would indicate previously unseen blazar physics, but the broadband data presented in this paper currently do not support such a conclusion.

In conclusion we find 1ES 1101-232 to be at an extreme end of blazar characteristics. This is the first time a detected blazar 
spectrum peaks above the VHE band. The data challenge current blazar models in the high-energy end of the electromagnetic spectrum.

Acknowledgements. The support of the Namibian authorities and of the University of Namibia in facilitating the construction and operation of HESS is gratefully acknowledged, as is the support by the German Ministry for Education and Research (BMBF), the Max Planck Society, the French Ministry for Research, the CNRS-IN2P3 and the Astroparticle Interdisciplinary Programme of the CNRS, the UK Particle Physics and Astronomy Research Council (PPARC), the IPNP of the Charles University, the South African Department of Science and Technology and National Research Foundation, and by the University of Namibia. We appreciate the excellent work of the technical support staff in Berlin, Durham, Hamburg, Heidelberg, Palaiseau, Paris, Saclay, and in Namibia in the construction and operation of the equipment.

We thank the ROTSE collaboration for providing the ROTSE $3 \mathrm{c}$ optical data, and L. Ostorero for help with the optical data analysis. This research has made use of the NASA/IPAC Extragalactic Database (NED). The R-band image around 1ES 1101-232, taken with the UK Schmidt telescope, was retrieved from the digital sky survey provided by the ST ScI. This work uses data obtained at the Nançay Radio Telescope.

\section{References}

Abraham, R. G., McHardy, I. M., \& Crawford, C. S. 1991, MNRAS, 252, 482 Aharonian, F. A. 2000, New Astron., 5, 377

Aharonian, F. A. 2001, Invited, Rapporteur, and Highlight Papers, Proc. 27th

ICRC (Hamburg), ed. R. Schlickeiser, 250 [arXiv : astro-ph/0112314]

Aharonian, F. A., et al. (The HEGRA collaboration) 1999, A\&A, 342, 69

Aharonian, F. A., Timokhin, A. N., \& Plyasheshnikov, A. V. 2002a, A\&A, 384, 834

Aharonian, F. A., et al. (The HEGRA collaboration) 2002b, A\&A, 393, 89

Aharonian, F. A., et al. (HESS Collaboration) 2005a, A\&A, 442, 895

Aharonian, F. A., et al. (HESS Collaboration) 2005b, A\&A, 430, 865

Aharonian, F. A., et al. (HESS Collaboration) 2006a, Nature, 440, 1018 (AHA06a)

Aharonian, F. A., et al. (HESS Collaboration) 2006b, A\&A, 455, 461

Aharonian, F. A., et al. (HESS Collaboration) 2006c, A\&A, 457, 899

Akerlof, C. W., Kehoe, R. L., McKay, T. A., et al. 2003, PASP, 115, 132

Albert, J., Aliu, E., Anderhub, H., et al. 2006, ApJ, 639, 761

Band, D. L., \& Grindlay, J. E. 1986, ApJ, 308, 576

Benbow, W. 2005, for the HESS Collaboration, Towards a Network of

Atmospheric Cherenkov Detectors VII, ed. B. Degrange, \& G. Fontaine, 163 Bessel, M. S. 1979, PASP, 91, 589

Biermann, P. L., \& Strittmatter, P. A. 1987, ApJ, 322, 643

Blackburn, J. K. 1995, ASP Conf. Ser., 77, ed. R. A. Shaw, H. E. Payne, \& J. J. E. Hayes

Błażejowski, M., Blaylock, G., \& Bond, I. H. 2005, ApJ, 630, 130

Buckley, D. A. H., Tuhoy, I. R., \& Remillard, R. A. 1985, Proc. ASA, 6, 147

Cardelli, J. A., Clayton, G. C., \& Mathis, J. S. 1989, ApJ, 345, 245

Chadwick, P. M., Lyons, K., McComb, T. J. L., et al. 1999, ApJ, 521, 547

Coppi, P. S., \& Aharonian, F. A. 1999, ApJ, 521, L33

Costamante, L., \& Ghisellini, G. 2002, A\&A, 384, 56

Dermer, C. D., \& Schlickeiser, R. 1993, ApJ, 416, 484

Donato, D., Ghisellini, G., Tagliaferri, G., \& Fossati, G. 2001, A\&A, 375, 739

Donato, D., Sambruna, R. M., \& Gliozzi, M. 2005, A\&A, 433, 1163

Douglas, J. N., Bash, F. N., Bozyan, F. A., et al. 1996, AJ, 111, 1945

Elvis, M., Plummer, D., Schachter, J., \& Fabbiano, G. 1992, ApJS, 80, 257

Falomo, R., \& Ulrich, M.-H. 2000, A\&A, 357, 91

Falomo, R., Bersanelli, M., Bouchet, P., \& Tanzi, E. G. 1993, AJ, 106, 11

Falomo, R., Scarpa, R., \& Bersanelli, M. 1994, ApJS, 93, 125

Fossati, G., Celotti, A., Chiaberge, M., et al. 2000, ApJ, 541, 166

Fukugita, M., Shimasaku, K., \& Ichikawa, T. 1995, PASP, 107, 945

Ghisellini, G., Maraschi, L., \& Treves, A. 1985, A\&A, 146, 204

Ghisellini, G., Celotti, A., \& Costamante, L. 2002, A\&A, 386, 833

Griffith, M. R., Wright, A. E., Burke, B. F., \& Ekers, R. D. 1994, ApJS, 90, 179

Henri, G., \& Pelletier, G. 1991, ApJ, 383, L7

Hinton, J. A., et al. (HESS Collaboration) 2004, New Astron. Rev., 48, 331

Hinton., J., Berge, D., Funk, S., \& the HESS Collaboration 2005, Towards a

Network of Atmospheric Cherenkov Detectors VII, ed. B. Degrange, \& G. Fontaine, 183

Kardashev, N. S. 1962, Sov. Astron., 6, 317

Katarzyński, K., Sol, H., \& Kus, A. 2001, A\&A, 367, 809
Katarzyński, K., Ghisellini, G., Tavecchio, F., et al. 2006, MNRAS, 368, 52 Kirsch, M., et al. 2006, available online at

http://xmm.vilspa.esa.es/docs/documents/CAL-TN-0018.pdf

Kneiske, T. M., Bretz, T., Mannheim, K., \& Hartmann, D. H. 2004, A\&A, 413, 807

Krawczynski, H., Coppi, P. S., \& Aharonian, F. A. 2002, MNRAS, 336, 721

Krawczynski, H., Hughes, S. B., Horan, D., et al. 2004, ApJ, 601, 151

Krennrich, F. Bond, I. H., Bradbury, S. M., et al. 2002, ApJ, 575, L9

Laurent-Muehleisen, S. A., Kollgaard, R. I., Moellenbrock, G. A., \& Feigelson, E. D. 1993, AJ, 106, 875

Lemoine-Goumard, M., Degrange, B., \& Tluczykont, M. 2006, Astropart. Phys., 25,195

Lin, Y. C., Bertsch, D. L., Dingus, B. L., et al. 1996, A\&AS, 120, 499

Maccagni, D., Tarenghi, M., Cooke, B. A., et al. 1978, A\&A, 62, 127

Madau, P., \& Pozzetti, L. 2000, MNRAS, 312, L9

Madejski, G. M., \& Schwartz, D. A. 1983, ApJ, 275, 467

Malkov, M. A., \& Drury, L. O'C., et al. 2001, Rep. Prog. Phys., 64, 529

McHardy, I. M., Lawrence, A., Pye, J. P., \& Pounds, K. A. 1981, MNRAS, 197, 893

Molendi, S., \& Sembay, S. 2003, available online at

http://xmm.vilspa.esa.es/docs/documents/

CAL-TN-0036-1-0.ps.gz

Monet, D., Bird A., Canzian, B., et al. 1998, available online at http://vizier.cfa.harvard.edu/vizier/VizieR/pmm/usno2.htx

Mücke, A., \& Protheroe, R. J. 2001, Astropart. Phys., 15, 121

Mushotzky, R. F. 1977, Nature, 265, 225

Ong, R. 2005, Invited, Rapporteur, and Highlight Papers, Proc. 29th ICRC (Pune), 10, 329 [arXiv: astro-ph/0605191]

Perlman, E. S., Stocke, J. T., Schachter, J., et al. 1996, ApJS, 104, 251

Perlman, E. S., Madejski, G., Georganopoulos, M., et al. 2005, ApJ, 625, 727

Pesce, J. E., Falomo, R., \& Treves, A. 1994, AJ, 107, 494

Pinkau, K. 1980, A\&A, 87, 192

Pohl, M., \& Schlickeiser, R. 2000, A\&A, 354, 395

Primack, J. R., Somerville, R. S., Bullock, J. S., \& Devriendt, J. E. G. 2001, Probing Galaxy formation with high energy gamma rays, AIP Conf. Proc., 558,463

Primack, J. R., Bullock, J. S., \& Somerville, R. S. 2005, Observational Gammaray Cosmology, AIP Conf. Proc., 745, 23

Remillard, R. A., Tuhoy, I. R., Brissenden, R. J. V., et al. 1989, ApJ, 345, 140

Romero, G. E., Cellone, S. A., \& Combi, J. A. 1999, ApJS, 135, 477

Saugé, L., \& Henri, G. 2004, ApJ, 616, 136

Scarpa, R., \& Falomo, R. 1997, A\&A, 325, 109

Schlegel, D. J., Finkbeiner, D. P., \& Davis, M. 1998, ApJ, 500, 525

Schlickeiser, R. 1985, A\&A, 143, 431

Sikora, M., Begelmann, M. C., \& Rees, M. J. 1994, ApJ, 421, 153

Sikora, M., Madejski, G., Moderski, R., \& Poutanen, J. 1997, ApJ, 484, 108

Smith, P. S., Jannuzi, B. T., \& Elston, R. 1991, ApJS, 77, 67

Stecker, F. W., Malkan, M. A., \& Scully, S. T. 2006, ApJ, 648, 774

Stecker, F. W., \& Scully, S. T. 2006, ApJ, 652, L9

Totani, T., Yoshii, Y., Iwamuro, F., et al. 2001, ApJ, 550, L137

Wilms, J., Allen, A., \& McCray, R. 2000, ApJ, 542, 914

Wolter, A., Comastri, A., Ghisellini, G., et al. 1998, A\&A, 335, 899

Wolter, A., Tavecchio, F., Caccianiga, A., Ghisellini, G., \& Tagliaferri, G. 2000, A\&A, 357, 429

Xue, Y., \& Cui, W. 2005, ApJ, 622, 160

Zacharias, N., Urban, S. E., Zacharias, M. I., et al. 2004, AJ, 127, 3043

1 Max-Planck-Institut für Kernphysik, PO Box 103980, 69029 Heidelberg, Germany

2 Yerevan Physics Institute, 2 Alikhanian Brothers St., 375036

Yerevan, Armenia

3 Centre d'Étude Spatiale des Rayonnements, CNRS/UPS, 9 av. du Colonel Roche, BP 4346, 31029 Toulouse Cedex 4, France

4 Universität Hamburg, Institut für Experimentalphysik, Luruper Chaussee 149, 22761 Hamburg, Germany

5 Institut für Physik, Humboldt-Universität zu Berlin, Newtonstr. 15, 12489 Berlin, Germany

${ }^{6}$ LUTH, UMR 8102 du CNRS, Observatoire de Paris, Section de Meudon, 92195 Meudon Cedex, France

7 DAPNIA/DSM/CEA, CE Saclay, 91191 Gif-sur-Yvette, Cedex, France

8 University of Durham, Department of Physics, South Road, Durham DH1 3LE, UK 
9 Unit for Space Physics, North-West University, Potchefstroom 2520, South Africa

${ }^{10}$ Laboratoire Leprince-Ringuet, IN2P3/CNRS, École Polytechnique, 91128 Palaiseau, France

11 Laboratoire d'Annecy-le-Vieux de Physique des Particules, IN2P3/CNRS, 9 Chemin de Bellevue, BP 110, 74941 Annecy-leVieux Cedex, France

12 APC, 11 place Marcelin Berthelot, 75231 Paris Cedex 05, France UMR 7164 (CNRS, Université Paris VII, CEA, Observatoire de Paris)

13 Dublin Institute for Advanced Studies, 5 Merrion Square, Dublin 2, Ireland

${ }^{14}$ Landessternwarte, Universität Heidelberg, Königstuhl, 69117 Heidelberg, Germany

e-mail: G.Puehlhofer@lsw.uni-heidelberg.de

${ }^{15}$ Laboratoire de Physique Théorique et Astroparticules, IN2P3/CNRS, Université Montpellier II, CC 70, Place Eugène Bataillon, 34095 Montpellier Cedex 5, France
${ }^{16}$ Universität Erlangen-Nürnberg, Physikalisches Institut, ErwinRommel-Str. 1, 91058 Erlangen, Germany

17 Laboratoire d'Astrophysique de Grenoble, INSU/CNRS, Université Joseph Fourier, BP 53, 38041 Grenoble Cedex 9, France

18 Institut für Astronomie und Astrophysik, Universität Tübingen, Sand 1, 72076 Tübingen, Germany

${ }^{19}$ Laboratoire de Physique Nucléaire et de Hautes Énergies, IN2P3/CNRS, Universités Paris VI \& VII, 4 place Jussieu, 75252 Paris Cedex 5, France

${ }^{20}$ Institute of Particle and Nuclear Physics, Charles University, V Holesovickach 2, 18000 Prague 8, Czech Republic

${ }^{21}$ Institut für Theoretische Physik, Lehrstuhl IV: Weltraum und Astrophysik, Ruhr-Universität Bochum, 44780 Bochum, Germany

${ }_{22}$ University of Namibia, Private Bag 13301, Windhoek, Namibia

23 European Associated Laboratory for Gamma-Ray Astronomy, jointly supported by CNRS and MPG 